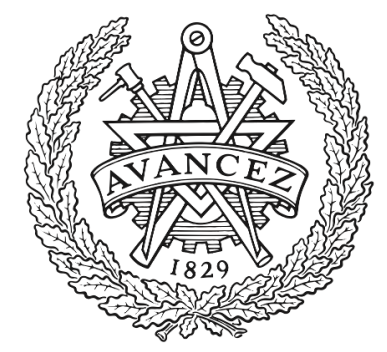

CHALMERS

UNIVERSITY OF TECHNOLOGY

\title{
Multibody dynamic modelling of a direct wind turbine drive train
}

Downloaded from: https://research.chalmers.se, 2023-04-26 09:31 UTC

Citation for the original published paper (version of record):

Asadi, S., Johansson, H. (2020). Multibody dynamic modelling of a direct wind turbine drive train.

Wind Engineering, 44(5): 519-547. http://dx.doi.org/10.1177/0309524X19849827

N.B. When citing this work, cite the original published paper. 


\title{
Multibody dynamic modelling of a direct wind turbine drive train
}

\author{
Saeed Asadi and Håkan Johansson \\ Chalmers University of Technology, Department of Mechanics and Maritime Sciences \\ SE-412 96 Gothenburg, Sweden \\ e-mails: saeed.asadi@chalmers.se, hakan.johansson@chalmers.se
}

\begin{abstract}
Wind turbines normally have a long operational lifetime and experience a wide range of operating conditions. A representative set of these conditions is considered as part of a design process, as codified in standards. However, operational experience shows that failures occur more frequently than expected, the more costly of these including failures in the main bearings and gearbox. As modern turbines are equipped with sophisticated online systems, an important task is to evaluate the drive train dynamics from online measurement data. In particular, internal forces leading to fatigue can only be determined indirectly from other locations' sensors. In this contribution, a direct wind turbine drive train is modelled using the floating frame of reference formulation for a flexible multibody dynamics system. The purpose is to evaluate drive train response based on blade root forces and bedplate motions. The dynamic response is evaluated in terms of main shaft deformation and main bearing forces under different wind conditions. The model was found to correspond well to a commercial wind turbine system simulation software (ViDyn).
\end{abstract}

Keywords: Wind turbine drive train dynamics, Flexible multibody dynamics, Floating frame of reference (FFR) formulation, Beam elements, Bearing damage index, Main shaft deflection.

\section{Introduction}

The demand for fossil free and renewable energy sources has boosted the wind power industry significantly in recent decades. To ensure economic growth and the sustainable future of the wind as energy source, one specific challenge is to reduce drive train failures in multi-MW horizontal axis wind turbines (HAWT), which are the predominant turbine type in terms of wind energy production. Despite the fact that wind turbines are designed and certified based on detailed predictions of the dynamic loads, there is still a need for improved modelling of the wind turbine drive train to understand the dynamic behavior and, thus, to gain new knowledge and, consequently, design more reliable drive lines [1].

This paper studies the HAWT drive train dynamic modelling to predict its behavior during operation and its implication on direct drive train components' fatigue life. The ultimate goal is to come closer to a tool that can evaluate measured turbine operational 
data in terms of drive train dynamics and its effect on drive train performance and components' fatigue life, based on blade root forces and moments, as well as bedplate motion. This analysis is crucial in evaluating different operation conditions. It can also be used as a priori to evaluate different proposed sensor positions.

Multibody dynamics techniques are commonly used to analyze the component loads in mechanical systems [2, 3]. Wind turbine drive trains could be considered to constitute a multibody system with flexible and rigid components interconnected with each other by kinematic constraints, and which experience force during different operating conditions. For instance, in [4], a flexible multibody dynamic system modelling is employed to study the dynamic response of a horizontal wind turbine gearbox composed of planetary and conventional parallel axis gear sets. In [5], the dynamic stability analysis of a horizontal axis wind turbine (HAWT) is presented. The analysis framework is established based on separation of the complete HAWT system model into rigid and flexible body subsystems. The most popular methods within flexible multibody dynamics modelling are floating frame of reference (FFR) formulation [2, 6, 7, 8, 9], absolute nodal coordinates formulation (ANCF) [10, 11], and corotational frame of reference (CFR) [12, 13].

The FFR formulation is based on separating the overall motion of the elastic body into a large rigid body motion (or reference motion), and superimposing small deformations by introducing a body fixed coordinate system, which translate and rotate with the body and experience small deformations (measured relative to this coordinate system). In [10], the traditional FFR approach combined with linear elastic finite element (FE) models is illustrated. In contrast, the ANCF does not separate the total elastic body motion into a reference motion and elastic deformations relative to the reference motion [10]. Consequently, ANCF leads to nonlinear expressions for the elastic forces and, in general, linear expressions for the inertia terms. Moreover, the element nodal coordinates are defined in the inertial frame used with a global shape function, and have a complete set of rigid body modes $[14,15,16]$. Thus, the global position vector of an arbitrary point on the element could be described by applying the global shape function and the absolute nodal coordinates. Another advantage of ANCF is that it is convenient when the deformations within the body are not small, for instance, in the case of long and slender wind turbine blades.

Comparing FFR with CFR, the major advantage of FFR is that it allows model order reduction, whereby the local generalized coordinates can be expressed as a linear combination of a small number of eigenmodes [17].

The multibody system simulation becomes computationally expensive by introducing a large number of degrees of freedom (DOF) upon FE discretization of elastic bodies within the FFR approach, especially when the internal forces are nonlinear. Thus, the reduction of elastic DOF is essential. In [18, 10], MOR methods have been applied in the FFR formulation. One approach is to select specific low-frequency bending vibration modes, besides torsional and axial modes, and to include them in the reduction basis and consequently, for the nonlinear response [19]. However, the fundamental axial modes' frequencies extraction of such modes is difficult and expensive, since they are much higher than the frequencies of the bending modes $[20,21]$.

Since the goal is to evaluate the drive train over long periods of time and under different operating conditions, a set of objective functions are defined. These functions are measures evaluated from the time-series solutions of the mathematical model. The purpose of these measures is that they can be used to quantify some aspects of the drive train system dynamics' behavior. In this study, the objective functions chosen are estimated damage in front and rear main bearings, and motion of main shaft at rotor hub and generator.

In the current study of the system dynamics model, the bearing forces causing the bearing damage, and the deflection fields, representing the system dynamics, are in focus. The outcome of the current research contributes to modelling wind turbine drive train dynamics' behavior in different operational scenarios, quantifying the effects on the drive train objective functions such as bearing forces and damage index, and could be applied as a methodology to contribute to virtual condition monitoring in order to detect and predict faults, and prevent failures in early stages in different components of drive trains.

The paper is outlined as follows: First, the multibody formalism of a direct drive wind turbine is presented. Particular attention is given to the parameterization of the main shaft flexibility. Afterwards, the comparison between the full and simplified (without flexible modes) mathematical models is demonstrated with respect to defined output functions (deflection fields and bearing forces). Also, a comparison with another simulation tool, ViDyn, is made. The drive train dynamics are quantified by suitably defined output functions related to tip deflection and bearing forces, allowing the different model assumptions and operating conditions to be assessed. 


\section{Multibody formulation of direct drive train wind turbine}

\subsection{Model description}

The structure of the studied direct drive wind turbine is shown in Fig. 1. The drive line includes the flexible main shaft with one inertia at each end, representing the rotor inertia and the generator inertia, respectively. The main shaft passes through two main bearings which are connected to the bedplate. The weight of the generator is carried by the main shaft while the generator counter-torque is transferred via a torque arm to the bedplate. The main shaft $(\mathrm{ms})$ is considered to be a separate flexible body within the multibody dynamics formalism, while the bedplate including the stator is assumed to be one rigid body ( $b p$ ). The bearings including housing flexibilities, are modelled as point flexibilities.
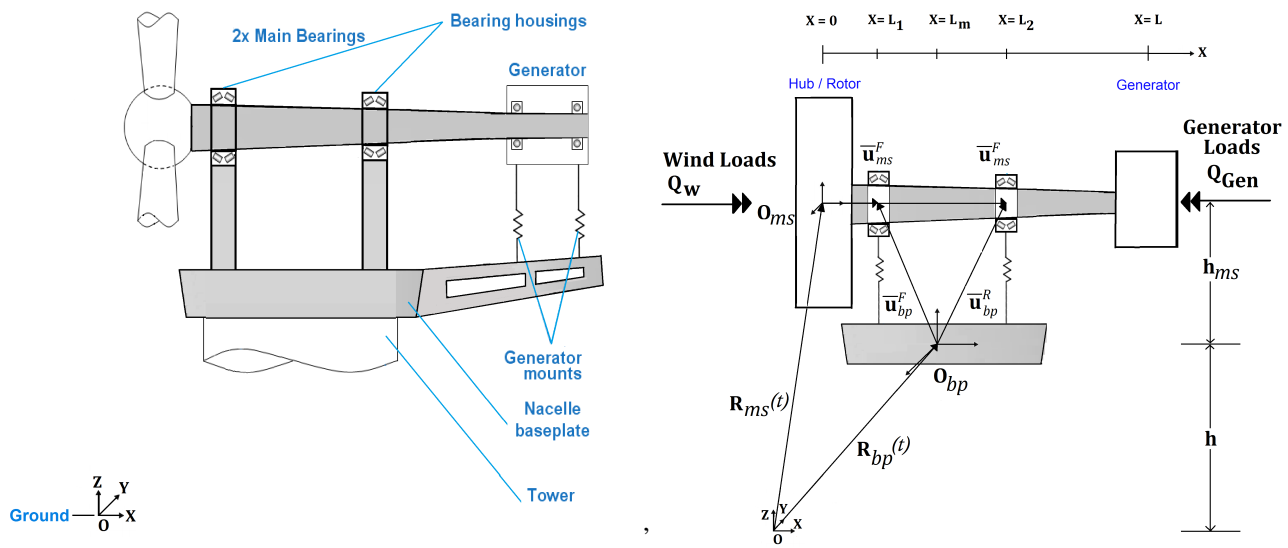

Fig. 1 Illustration of studied direct drive wind turbine (left) engineering model (right)

Following floating point of reference formulation ([23]), the position of a point $P$ on the bedplate is expressed as:

$$
\mathbf{r}_{b p}^{P}=\mathbf{R}_{b p}+\mathbf{A}\left(\theta_{b p}\right) \overline{\mathbf{u}}_{b p}^{P}
$$

where $\mathbf{R}$ is the position of the body fixed frame, $\mathbf{A}$ is the body fixed rotation matrix and $\overline{\mathbf{u}}$ is the position vector in the body fixed frame. Correspondingly, a point $Q$ on the main shaft is expressed as:

$$
\mathbf{r}_{m s}^{Q}=\mathbf{R}_{m s}+\mathbf{A}\left(\theta_{m s}\right) \overline{\mathbf{u}}_{m s}^{Q}=\mathbf{R}_{m s}+\mathbf{A}\left(\theta_{m s}\right)\left(\overline{\mathbf{u}}_{m s_{Q}}^{0}+\mathbf{S}\left(\mathbf{x}_{Q}\right) \mathbf{q}_{m s}\right)
$$

such that the flexibility of the main shaft is parameterized in terms of a space-dependent shape matrix $\mathbf{S}$ and a vector of a timedependent elastic generalized coordinates $\mathbf{q}_{m s s}$. By adopting $\theta_{m s}$ and $\theta_{b p}$ as Euler angles parameterization of rotation matrix $\mathbf{A}$, the motion of the considered system is described by the set of time-dependent variables $\mathbf{q}$, defined as:

$$
\mathbf{q}=\left[\begin{array}{lll}
\underbrace{\mathbf{R}_{m s}^{T}, \theta_{m s}^{T},}_{\mathbf{q}_{m s}^{T}} \mathbf{q}_{m s}^{T}, & \underbrace{\mathbf{R}_{b p}^{T}, \theta_{b p}^{T}}_{\mathbf{q}_{b p}^{T}}
\end{array}\right]^{T}
$$

Since it can be expected that the rotation around the $Y$-axis will be small, the Bryant " $X-Y-Z$ " convention of Euler angles is adopted in contrast to the " $Z-X-Z$ " classical Euler convention to avoid the problem of singularity [24].

Below, the drive train system performance is studied under prescribed bedplate motion, in which case $\mathbf{R}_{b p}$ and $\theta_{b p}$ are given 
functions of time. Hence the set of degrees of freedom for the considered system is collected in the vector $\mathbf{q}_{m s}$ as:

$$
\mathbf{q}_{m s}=\left[\begin{array}{lll}
\mathbf{R}_{m s}^{T}, & \theta_{m s}^{T}, & \mathbf{q}_{f m s}^{T}
\end{array}\right]^{T}
$$

\subsection{Equation of motion}

Following [23], the principal of virtual work is here expressed as $\delta W_{i}=\delta W_{e}$ where $\delta W_{i}$ is the virtual work from inertia forces and $\delta W_{e}$ is the virtual work from applied forces. The applied forces are separated into conservative $\delta W_{c}$ and non-conservative $\delta W_{n c}$ virtual work. In order to obtain a formulation suitable for numerical implementation using an explicit time-stepping algorithm, we follow [23] and split $\delta W_{i}$ into two parts as

$$
\delta W_{i}=\left(\frac{d}{d t}\left(\frac{\partial T}{\partial \dot{\mathbf{q}}}\right)^{T}-\left(\frac{\partial T}{\partial \mathbf{q}}\right)^{T}\right) \delta \mathbf{q}=[\underbrace{\mathbf{M} \ddot{\mathbf{q}}-\dot{\mathbf{M}} \dot{\mathbf{q}}+\left(\frac{\partial T}{\partial \mathbf{q}}\right)^{T}}_{-\mathbf{Q}_{v}}]^{T} \delta \mathbf{q}
$$

where $T$ is the system kinetic energy (cf. sec. 2.3), $\mathbf{M}$ is a system mass matrix and $\mathbf{Q}_{v}$ is the force vector of centrifugal and Coriolis forces. The virtual work from applied forces is expressed as

$$
\delta W_{e}=\delta W_{c}+\delta W_{n c}=\underbrace{\delta W_{e}+\delta W_{b}+\delta W_{g}}_{\delta W_{c}}+\delta W_{n c}=\left(\mathbf{Q}_{c}+\mathbf{Q}_{e x t}\right) \delta \mathbf{q}=\left(\mathbf{Q}_{e}+\mathbf{Q}_{b}+\mathbf{Q}_{g}+\mathbf{Q}_{e x t}\right) \delta \mathbf{q}
$$

where $\mathbf{Q}_{c}\left(=\mathbf{Q}_{e}+\mathbf{Q}_{b}+\mathbf{Q}_{g}\right)$ are the forces due to elastic deformation of the main shaft $\left(\mathbf{Q}_{e}\right)$ and the bearings mounting $\left(\mathbf{Q}_{b}\right)$ and $\mathbf{Q}_{\text {ext }}$ is externally applied forces. Eq. (5) and Eq. (6) give rise to the equation of motion on matrix form:

$$
\mathbf{M} \ddot{\mathbf{q}}_{m s}=\mathbf{Q}_{e x t}\left(\mathbf{q}_{m s}\right)+\mathbf{Q}_{e}\left(\mathbf{q}_{m s}\right)+\mathbf{Q}_{b}\left(\mathbf{q}_{m s}, \mathbf{q}_{b p}\right)+\mathbf{Q}_{g}+\mathbf{Q}_{v}\left(\mathbf{q}_{m s}\right)
$$

The numerical simulation is performed using a variable-order time-stepping method as implemented in the function ode $15 \mathrm{~s}$ in Matlab R2013b.

\subsection{Inertia forces}

The kinetic energy of the system can be written as

$$
T=T^{m s}+T^{b p} .
$$

Upon considering the parameterization of material points of the main shaft (Eq. (2)), kinetic energy related to the main shaft is:

$$
T^{m s}=\frac{1}{2} \int_{V} \rho\left(\dot{\mathbf{r}}_{m s}\right)^{T} \dot{\mathbf{r}}_{m s} d V=\frac{1}{2} \dot{\mathbf{q}}_{m s}^{T} \mathbf{M} \dot{\mathbf{q}}_{m s}
$$

Using the definition of kinetic energy $T^{m s}$ and the mass matrix $\mathbf{M}$, the quadratic velocity centrifugal and Coriolis force vector $\mathbf{Q}_{v}$ can be expressed from the Lagrange equation in a closed form as indicated by Eq. (5), as detailed in App. A, in Eq. (32) and Eq. (61). The specific formulation of the velocity-dependent inertia forces was based on [22], which was shown to require less arithmetic operations than the formulation of Shabana [23].

\subsection{Elastic forces}

In this section, the formulation of the generalized applied forces is briefly stated. This includes the main shaft deformation due to elastic forces, the bearing forces, and the gravity.

\subsubsection{Main shaft deformation}

The virtual work from elastic forces of the main shaft considering bending, elongation, and torsion under an assumption of small strains, is given as

$$
\delta W_{e}=-\delta U
$$


where the shaft is assumed to be adequately described by the Euler-Bernoulli theory as

$$
U=\frac{1}{2} \int_{0}^{L}\left[E I_{z}\left(\frac{\partial^{2} w_{Y}}{\partial x^{2}}\right)^{2}+E I_{y}\left(\frac{\partial^{2} w_{Z}}{\partial x^{2}}\right)^{2}+E A\left(\frac{\partial u_{X}}{\partial x}\right)^{2}+G J\left(\frac{\partial u_{T}}{\partial x}\right)^{2}\right] d x=\frac{1}{2} \mathbf{q}_{f_{m s}^{T}}^{T} \mathbf{K}_{f f}^{m s} \mathbf{q}_{f_{m s}}
$$

where $\mathbf{K}_{f f}^{m s}$ is a symmetric positive definite stiffness matrix associated with the elastic coordinates of the main shaft. The detailed derivation of $\mathbf{K}_{f f}^{m s}$ definition is presented in App. B (Eq.(39)-(40)). By introducing material damping matrix $\mathbf{C}_{f f}$, the main shaft force vector $\mathbf{Q}_{e}$ becomes:

$$
\mathbf{Q}_{e}=-\left(\mathbf{K}_{f f}^{m s} \mathbf{q}_{f}^{m s}+\mathbf{C}_{f f}^{m s} \dot{\mathbf{q}}_{f}^{m s}\right)
$$

\subsubsection{Bearing generalized forces}

The virtual work for bearing and bearing housing deformation is obtained from

$$
\delta W_{b}=-\delta U_{b}^{F}-\delta U_{b}^{R}
$$

where

$$
U_{b}^{i}=\frac{1}{2} K_{B H_{X}} l_{X}^{i^{2}}+\frac{1}{2} K_{B H_{Y}} l_{Y}^{i^{2}}+\frac{1}{2} K_{B H_{Z}} l_{Z}^{i^{2}}, i=\{F, R\}
$$

for the front (F) and rear (R) bearings respectively. Here, $l_{X}^{i}, l_{Y}^{i}, l_{Z}^{i}$ are the bearing deformation expressed by the difference of the bearings' location in the main shaft and the bedplate coordinate, i.e

$$
\mathbf{l}^{i}=\left(\mathbf{R}_{m s}+\mathbf{A}\left(\theta_{m s}\right) \overline{\mathbf{u}}_{m s}^{i}\right)-\left(\mathbf{R}_{b p}+\mathbf{A}\left(\theta_{b p}\right) \overline{\mathbf{u}}_{b p}^{i}\right)
$$

where $i$ denotes the location of the bearing in front $(i=F)$ or rear $(i=R)$ according to Fig. 1. The derivation of generalized force vector $\mathbf{Q}_{b}^{i}$ associated with bearing deformation and damping is given in App. 6.4.

\subsubsection{Gravity}

The virtual work for gravity is obtained from:

$$
\delta W_{g}=-\delta U_{g}, \quad \text { where } \quad U_{g}=\int_{V} \rho r_{z} g d V
$$

which gives $\mathbf{Q}_{g}$ as

$$
\mathbf{Q}_{g}^{i}=\mathbf{M}^{i} \mathbf{g}^{T}
$$

where $\mathbf{M}$ is the mass matrix, and $i$ denotes rotor, generator, and the main shaft, and $\mathbf{g}(=[0,0,-g, 0,0,0,0,0,0,0,0,0])$ is the gravity vector.

\subsection{Main shaft flexibility parameterization}

In order to construct the shape function matrix $\mathbf{S}$, the eigenmodes of an Euler-Bernoulli beam with point mass inertia at ends and residing on two rigid supports are calculated considering bending, elongation and torsion. The main shaft includes the following structural parameters: $E, G, I, \rho, A, m_{m s}, m_{2}, m_{2}, L, L_{1}, L_{2}$.

For bending mode shapes, the following parameterization is chosen:

$$
m_{B_{i}}^{n}(x)=C_{1}^{i} \cos \left(\beta_{n} x\right)+C_{2}^{i} \sin \left(\beta_{n} x\right)+C_{3}^{i} \cosh \left(\beta_{n} x\right)+C_{4}^{i} \sinh \left(\beta_{n} x\right)
$$

where $i$ denotes the different deflection fields corresponding to the sides and middle part of the shaft $(i=1,2,3)$. $\beta_{n}$ refers to the $n^{\text {th }}$ bending eigenmode. $C_{1,2,3,4}^{i} \mathrm{~s}$ are obtained from the boundary conditions and continuity equations at bearing locations. The eigenvalues are calculated via the relationship $\omega_{n}=\beta_{n}^{2} c$, where $c=\sqrt{\frac{E I}{\rho A}}$.

For the axial elongation, considering that the front bearing is connected via a longitudinal spring along the shaft direction, there are 2 regions for elongation field $(i=1,2)$ parameterized by

$$
m_{X_{i}}^{n}(x)=C_{1}^{i} \cos \left(\lambda_{X}^{n} x\right)+C_{2}^{i} \sin \left(\lambda_{X}^{n} x\right)
$$


where $\lambda_{n}^{X}=\frac{\omega_{n}}{c_{E}}$ and $c_{E}=\sqrt{E / \rho}$.

For the torsional eigenmodes, the same type of parameterization is used:

$$
m_{T_{i}}^{n}(x)=C_{1}^{i} \cos \left(\lambda_{T}^{n} x\right)+C_{2}^{i} \sin \left(\lambda_{T}^{n} x\right)
$$

where $\lambda_{n}^{T}=\frac{\omega_{n}}{c_{S}}$ and $c_{S}=\sqrt{G / \rho}$.

The eigenmodes and eigenvalues are calculated using the Finite element method, and the respective coefficients $C_{i}$ in Eq. (18)- (20) are determined by curve fit. The bending, elongation and torsional mode shapes are shown in Fig. 2.
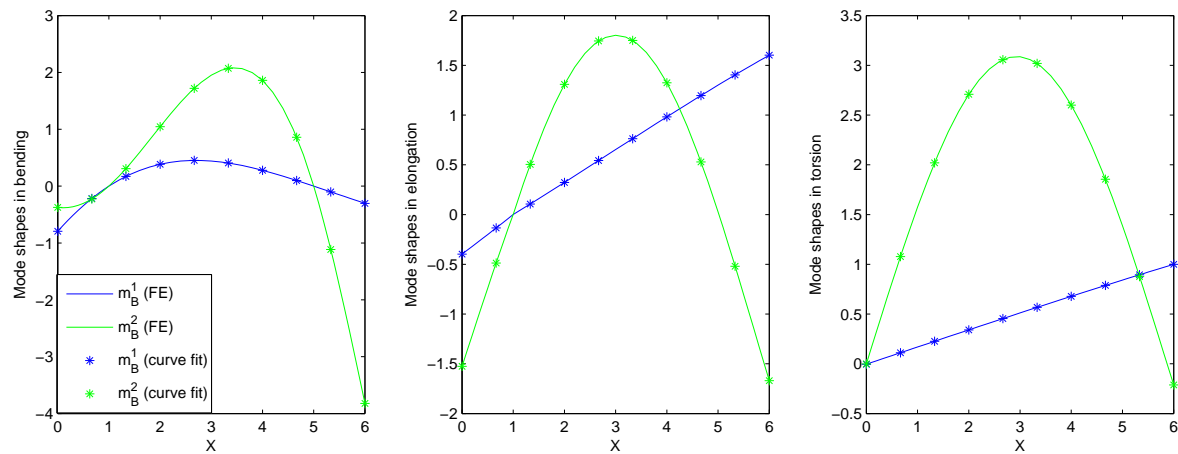

Fig. 2 The first $N_{m}=2$ Mode shapes for the beam element in bending (left), elongation (middle), and torsion (right) in both analytical and FE solution

Table. 1 shows the value of eigenmodes for the bending, elongation and torsional cases in both FE and analytical solutions:

Table 1 Eigenmodes for analytical and FE approaches

\begin{tabular}{lllll}
\hline \hline & \multicolumn{3}{c}{ FE solution } & \\
\cline { 2 - 4 } & Mode. 1 & Mode. 2 & $\begin{array}{l}1^{\text {st }} \text { Eigenfreq } \\
(\mathrm{Hz})\end{array}$ & $\begin{array}{l}2^{\text {nd }} \text { Eigenfreq } \\
(\mathrm{Hz})\end{array}$ \\
\hline Bending modes $\beta_{n}$ & 0.2156 & 0.4751 & 6.0816 & 29.5397 \\
Elongation modes $\lambda_{n}^{X}$ & 0.1591 & 0.5756 & 127.8008 & 462.4371 \\
Torsional modes $\lambda_{n}^{T}$ & 0.0760 & 0.5354 & 37.8392 & 266.7413 \\
\hline \hline
\end{tabular}

Upon checking the eigenfrequencies corresponding to stated modes, it is sufficient to consider the first two modes in the bending, and only the first modes in the torsion and elongation for the current study. It could also be demonstrated that the axial modes have higher frequencies even than rigid modes, thus they could be ignored. The advantage of having an analytical relation of the eigenmodes compared to FE shape functions is that the analytical relation could be utilized for the shape function by expressing them through defined mode shapes and higher order derivatives. The drawback of the current analytical approach in the main shaft is that it cannot capture the modes where there is motion in bearing locations, since the simply supported boundary conditions are assumed in these coordinates.

The elastic deformation due to elongation, torsion, and bending are expressed in terms of the shape functions as: 


$$
\begin{aligned}
& u_{X}(x) \approx m_{X}^{1}(x) q_{X}^{1}, \\
& u_{T}(x) \approx m_{T}^{1}(x) q_{T}^{1}, \\
& w_{Y}(x) \approx m_{B}^{1}(x) q_{Y}^{1}+m_{B}^{2}(x) q_{Y}^{2}, \\
& w_{Z}(x) \approx m_{B}^{1}(x) q_{Z}^{1}+m_{B}^{2}(x) q_{Z}^{2} .
\end{aligned}
$$

where $u_{X}, u_{T}, w_{Y}, w_{Z}$ and $q_{X}^{i}, q_{T}^{i}, q_{B_{Y}}^{i}, q_{B_{Z}}^{i}$ are displacement fields and DOF denotes the elongation, torsion and bending in $Y$ and $Z$ directions respectively. Here, it is assumed that it is sufficient to capture the main shaft flexibility using one mode for elongation and torsion, and two modes for bending. Hence, the shape matrix is obtained as:

$$
\overline{\mathbf{u}} \approx \underbrace{\left[\begin{array}{c}
X \\
Y \\
Z
\end{array}\right]}_{\mathbf{u}_{0}}+\underbrace{\left[\begin{array}{cccccc}
m_{X}^{1}(x) & 0 & Z\left(m_{B}^{1}(x)\right)^{\prime} & Z\left(m_{B}^{2}(x)\right)^{\prime} & -Y\left(m_{B}^{1}(x)\right)^{\prime} & -Y\left(m_{B}^{2}(x)\right)^{\prime} \\
0 & -Z m_{T}^{1}(x) & m_{B}^{1}(x) & m_{B}^{2}(x) & 0 & 0 \\
0 & Y m_{T}^{1}(x) & 0 & 0 & m_{B}^{1}(x) & m_{B}^{2}(x)
\end{array}\right]}_{\mathbf{S}(x)} \underbrace{\left[\begin{array}{c}
q_{X} \\
q_{T} \\
q_{B_{Y}}^{1} \\
q_{B_{Y}}^{2} \\
q_{B_{Z}}^{1} \\
q_{B_{Z}}^{2}
\end{array}\right]}_{\mathbf{q}_{f}}
$$

where $(\bullet)$ ' denotes derivatives with respect to $x$.

\subsection{Excitation}

The imposed excitation to the system is defined next. The excitation from wind is represented by a 6-component time-series consisting of the $3 \mathrm{D}$ force vector (in global frame) and the respective torque vector acting on the hub. In addition, there is a countertorque from the generator and the prescribed motion of the bedplate.

\subsubsection{Rotor loads}

The force and torque imposed on the hub is given as external excitation forces to the system. The external virtual work by force and torque components $\left(\mathbf{F}_{h u b}, \mathbf{T}_{h u b}\right)$ due to the wind loads at the hub $\left(X_{m s}=0\right)$, is defined as follows:

$$
\begin{aligned}
& \delta W_{F}=\mathbf{F}^{T} \delta \mathbf{r}_{h u b}=\mathbf{Q}_{R}^{F} \delta \mathbf{R}_{m s}+\mathbf{Q}_{\theta}^{F} \delta \theta_{m s}+\mathbf{Q}_{f}^{F} \delta \mathbf{q}_{f}, \\
& \delta W_{T}=\mathbf{T}^{T} \delta \Theta_{h u b}=\mathbf{Q}_{R}^{M} \delta \mathbf{R}_{m s}+\mathbf{Q}_{\theta}^{M} \delta \theta_{m s}+\mathbf{Q}_{f}^{M} \delta \mathbf{q}_{f} .
\end{aligned}
$$

where $\Theta_{h u b}=\Theta(0)$ and $\mathbf{r}_{h u b}=\left(\mathbf{R}_{m s}+\mathbf{A}\left(\theta_{m s}\right)\left(\overline{\mathbf{u}}_{m s}^{0}+\mathbf{S}(0) \mathbf{q}_{f}\right)\right)$. Note that the rotation matrix $\Theta$ including rigid rotations $\theta$ and the flexible components related to the rotation, is defined as follows:

$$
\Theta(0)=\left(\theta_{m s}+\mathbf{A}\left[\begin{array}{c}
u_{T}(0) \\
-W_{Z}^{\prime}(0) \\
W_{Y}^{\prime}(0)
\end{array}\right]\right)
$$

The detailed derivation of the aforementioned definitions is presented in App. 6.5.

\subsubsection{Generator excitation}

The generator excitation $T_{g e n}$ is imposed as axial rotation at the main shaft end at $X=L$ and is assumed to be proportional to the difference between a desired 'set' speed $\omega_{g}^{\text {set }}$ and the actual rotational speed of the shaft $\omega_{g}$ as

$$
T_{g e n}(t)=C_{g e n}\left(\omega_{g}^{s e t}-\omega_{m}\right)
$$


where $\omega_{m}$ denotes the rotational speed at shaft end, and $C_{g e n}=828.26 \mathrm{kNm} \mathrm{s} / \mathrm{rad}$.

$$
W_{T}(L)=\mathbf{T}^{T} \Theta_{g e n}
$$

where $\Theta_{\text {gen }}=\Theta(L)$.

and the corresponding virtual work done by $W_{M}(t)$ is:

$$
\delta W_{M}=\mathbf{Q}_{R}^{M} \delta \mathbf{R}_{m s}+\mathbf{Q}_{\theta}^{M} \delta \theta_{m s}+\mathbf{Q}_{f}^{M} \delta \mathbf{q}_{f}=T_{g e n}^{T} \delta \Theta_{g e n}
$$

The detailed derivation of the aforementioned definitions is presented in App. 6.6.

\section{Model adaptation and verification}

In the present study, the model is adapted to a multi-MW direct drive wind turbine. In order to test and verify the model under different turbine operating conditions, a system simulation model of the whole turbine, including wind field, blade aerodynamic loads, tower and control system was developed in ViDyn [27]. Simulations using this model were carried out for a number of turbulent wind fields following the IEC standard. From these simulations, the forces acting on the hub, the generator speed and bedplate motion were extracted and used as input in the mathematical model described in sec. 2 above. For model verification, the forces at bearings as well as shaft motion were also extracted from the ViDyn simulations. The parameters and their numerical values assumed in the mathematical model intended to be representative for a multi-Mw direct drive wind turbine are given in Table 3.

\begin{tabular}{lccc}
\hline \hline Tab. 2 Structural parameters for the direct drive train & & & \\
\hline \hline Parameter & Symbol & Value & Unit \\
\hline Total length of the main shaft in $X$ axis & $L$ & 6 & $\mathrm{~m}$ \\
Front bearing location in $X$ axis & $L_{1}$ & 1 & $\mathrm{~m}$ \\
Rear bearing location in $X$ axis & $L_{2}$ & 5 & $\mathrm{~m}$ \\
Bedplate height from the ground in $Z$ axis & $h$ & 80 & $\mathrm{~m}$ \\
Hub tilting angle from vertical $Z$ axis & $\theta_{y}^{H}$ & 4 & $\mathrm{deg}$ \\
Main shaft center line height from the bedplate center $O_{b p}$ & $h_{m s}$ & 0.8 & $\mathrm{~m}$ \\
Rotor hub mass & $m_{1}$ & 40000 & $\mathrm{~kg}$ \\
Generator rotor mass & $m_{2}$ & 30000 & $\mathrm{~kg}$ \\
Main shaft inertia mass & $m_{m s}$ & 1000 & $\mathrm{~kg}$ \\
Rotor equivalent radius & $R_{1}$ & 7 & $\mathrm{~m}$ \\
Generator equivalent radius & $R_{2}$ & 0.5 & $\mathrm{~m}$ \\
Main shaft radius & $R_{m s}$ & 0.3258 & $\mathrm{~m}$ \\
Generator inertia with respect to $X$ axis & $J_{g}$ & 0.087 & $\mathrm{~kg} \mathrm{~m}$ \\
Rotor inertia with respect to $X$ axis & $J_{r}$ & 0.095 & $\mathrm{~kg} \mathrm{~m}$ \\
Main shaft bending stiffness & $E I$ & 1000 & $\mathrm{MPa} \mathrm{m}$ \\
Main shaft front bearing longitudinal stiffness in $X$ direction & $K_{B}^{F}$ & 10000 & $\mathrm{MN} / \mathrm{m}$ \\
Main shaft front bearing longitudinal stiffness in $Y$ direction & $K_{B Y}^{F}$ & 3000 & $\mathrm{MN} / \mathrm{m}$ \\
Main shaft front bearing longitudinal stiffness in $Z$ direction & $K_{B Z}^{F}$ & 10000 & $\mathrm{MN} / \mathrm{m}$ \\
Main shaft rear bearing longitudinal stiffness in $X$ direction & $K_{B} R$ & 0 & $\mathrm{MN} / \mathrm{m}$ \\
Main shaft rear bearing longitudinal stiffness in $Y$ direction & $K_{B Y}^{R}$ & 3000 & $\mathrm{MN} / \mathrm{m}$ \\
Main shaft rear bearing longitudinal stiffness in $Z$ direction & $K_{B Z}^{R}$ & 10000 & $\mathrm{MN} / \mathrm{m}$ \\
Main shaft bearings longitudinal damping ratio & $\zeta$ & & $0.01 \mathrm{sec}$ \\
\hline \hline
\end{tabular}

\subsection{The full model and simplified model simulation responses}

As discussed earlier in sec. 2.1, the constructed mathematical model includes both rigid DOF ( $\mathbf{R}$ and $\theta$ ), and flexible DOF (q $f$ ). To compare the system dynamic response of a full mathematical model (including all aforementioned DOF) and a simplified model (considering only the rigid DOF), the hub and generator deflection time histories are evaluated as shown in Fig. 5. As can be seen, there is a strong correspondence between the two models, thus the simplified model response is quite close to the full model. 

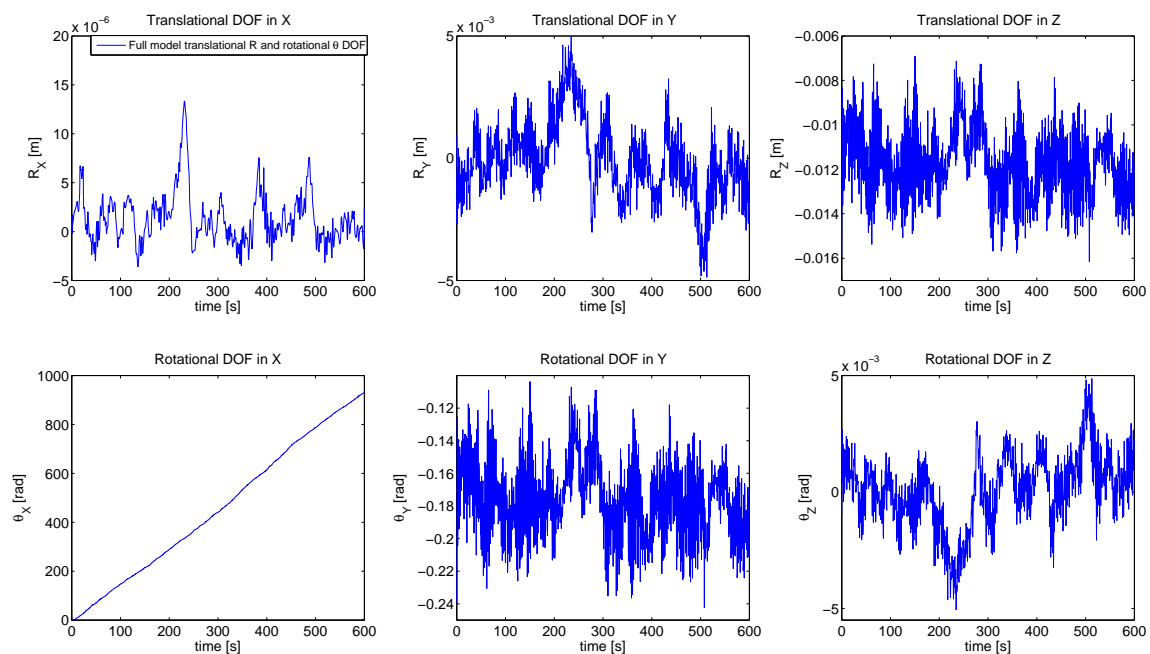

Fig. 3 The rigid DOF $\mathbf{q}_{m s}$, including the translational DOF $\mathbf{R}_{m s}$ (up), and the rotational DOF $\theta_{m s}$ (below), in the full model for mean wind speed of $11 \mathrm{~m} / \mathrm{s}$
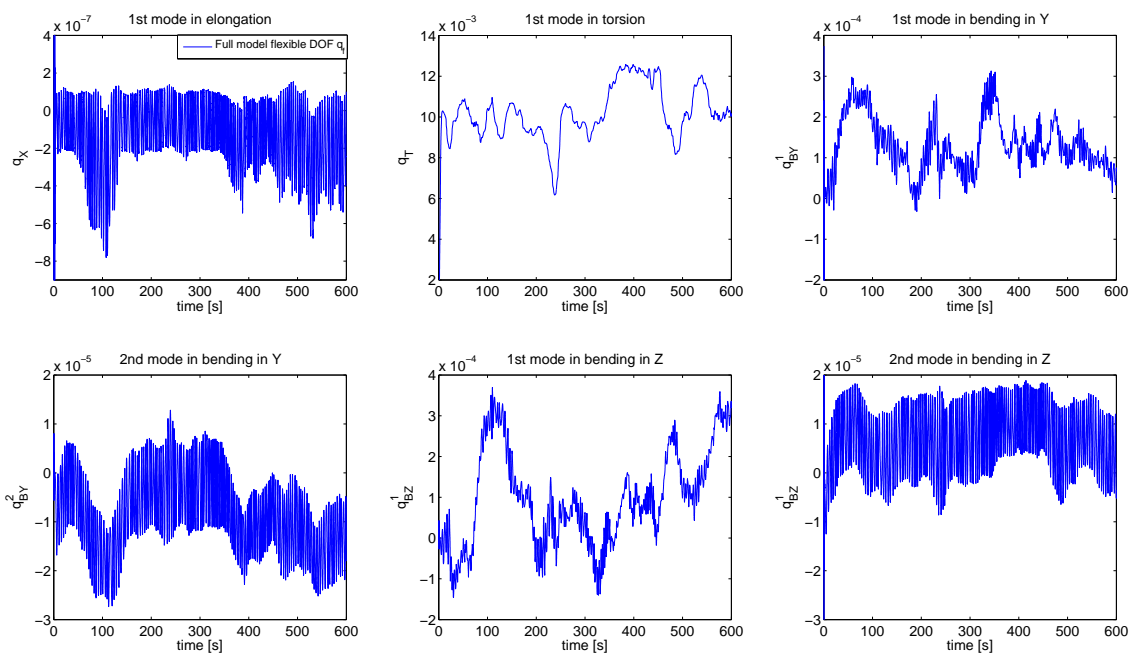

Fig. 4 The flexible DOF $\mathbf{q}_{m s}$ in the full model for mean wind speed of $11 \mathrm{~m} / \mathrm{s}$ 
As demonstrated in Fig. 4, it is noted that in the elongation mode, the range for $q_{X}$ is negligible compared to the other flexible DOF. This is somehow expectable, since the elongation modes have very minor effects in the system response here. It is also noted that the second modes in both bending DOF $\left(q_{B} y^{2}\right.$ and $\left.q_{B} z^{2}\right)$ have insignificant range compared to the first modes $\left(q_{B} y^{1}\right.$ and $\left.q_{B} z^{1}\right)$, since the dominant capture in the motion is abstracted mostly in the first modes. Moreover, the bending modes in $Y\left(q_{B_{Y}}^{1,2}\right)$ have similar behavior to $Z\left(q_{B_{Z}}^{1,2}\right)$, since they have the same mode shapes. High frequency behavior (especially in $\left.q_{B_{Y, Z}}^{2}\right)$ are seen in flexible DOF which could be ignored. Also the range of the behavior is generally small in these DOF.

The deflection fields at the rotor hub $(X=0)$ and the generator $(X=L)$ are shown in both full and simplified models.
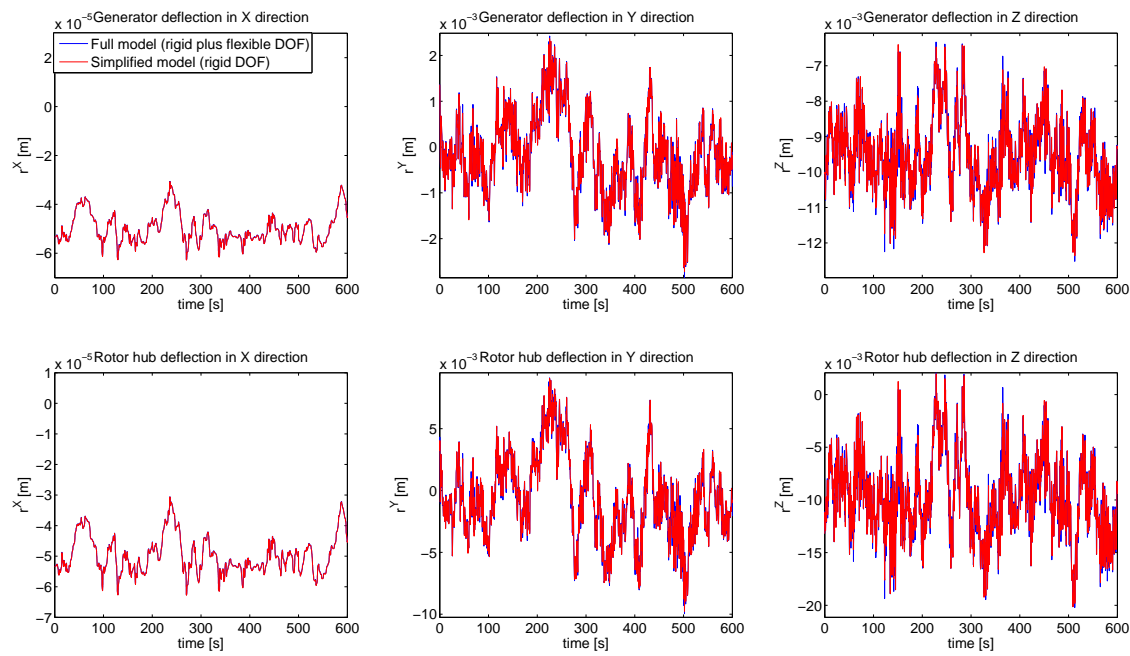

Fig. 5 The deflection fields at generator (up) and rotor hub (down), at $11 \mathrm{~m} / \mathrm{s}$, full mathematical model ——, and simplified model

\subsection{Model verification}

The current study considers several operational scenarios within a wind turbine community such as normal operation (with different wind speeds), turbulent cases, vertical inclination case, to evaluate the system dynamic responses in each scenario.

The system response is verified against the ViDyn model in terms of the deflection at the rotor hub and the generator (the endpoints of the main shaft), the front and rear bearing forces. Theses responses are shown for 3 different operational scenarios, corresponding to a turbine operating normally at mean wind speeds 6,11 and $18 \mathrm{~m} / \mathrm{s}$, respectively (turbulence intensity 0.12 ). In Figures Fig. 6- 11. namely "Normal operation" at different wind speeds $([6,11,18] \mathrm{m} / \mathrm{s})$. 
- Normal operation at mean wind speed $6 \mathrm{~m} / \mathrm{s}\left(O S^{1}\right)$ :

For the operational scenario $O S^{1}$, the system response in both the mathematical model and the ViDyn simulation model is illustrated in terms of deflections at the hub and the generator (Fig. 6), and the front and rear bearing forces in 3 directions (Fig. 7).
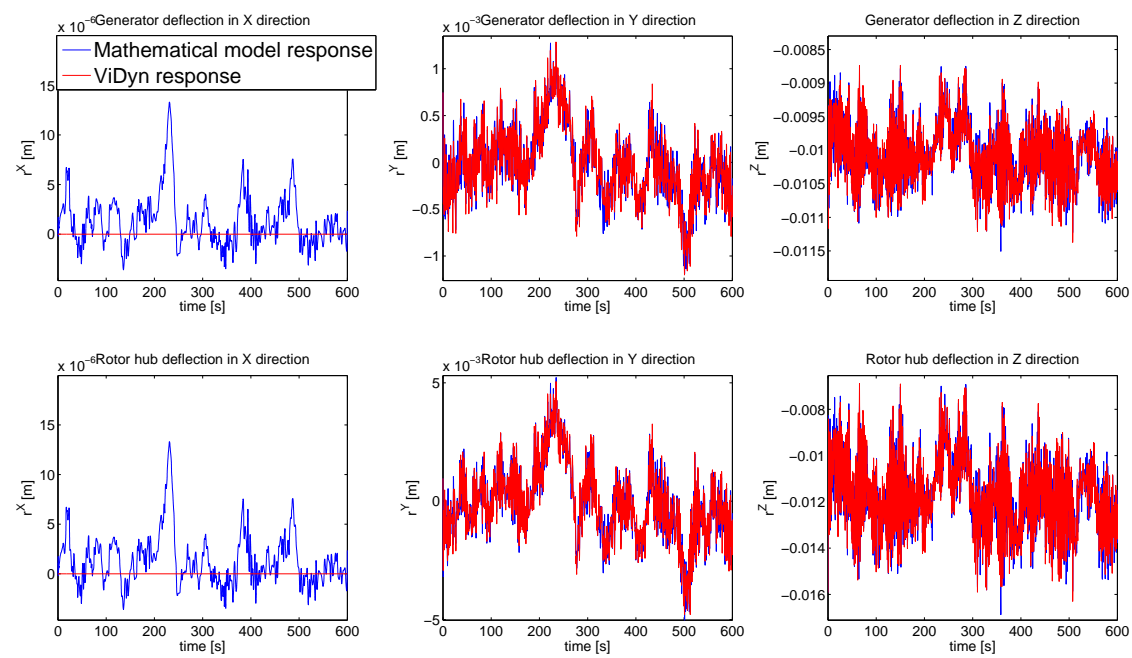

Fig. 6 The deflection fields at generator (up) and rotor hub (down), in $O S^{1}(6 \mathrm{~m} / \mathrm{s})$, Math and ViDyn models
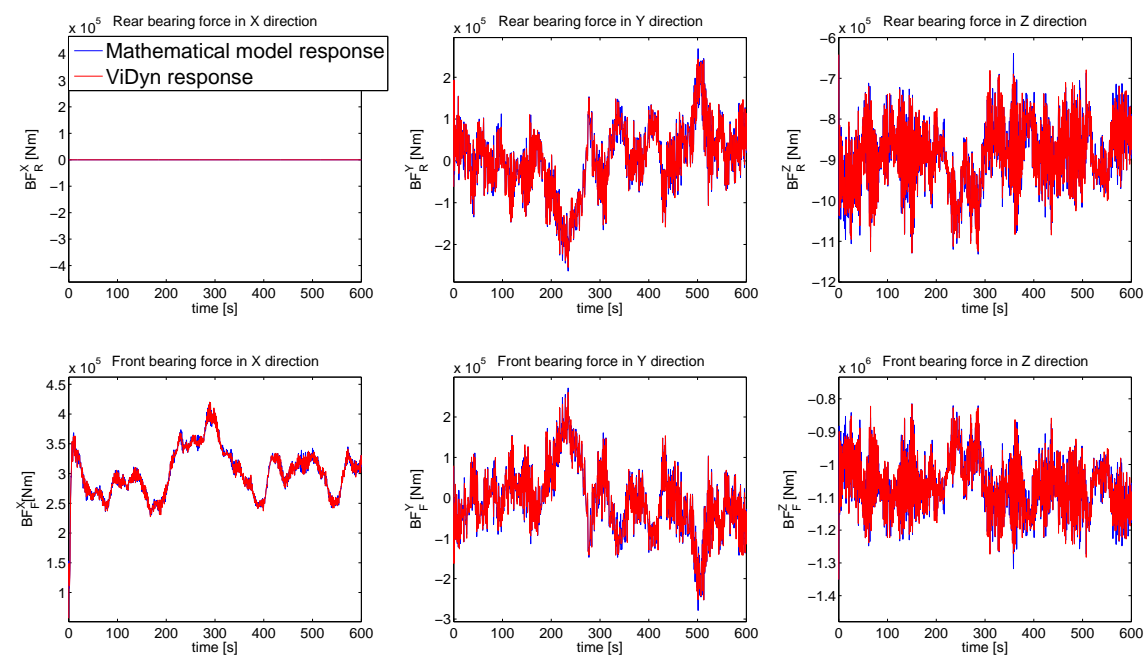

Fig. 7 The bearing forces in rear (up) and front (down), in $O S^{1}(6 \mathrm{~m} / \mathrm{s})$, Math , and ViDyn models 
- Normal operation at mean wind speed $11 \mathrm{~m} / \mathrm{s}\left(O S^{2}\right)$ :

For the operational scenario $O S^{2}$, the system response in both the mathematical model and the ViDyn simulation model is illustrated in terms of deflections at the hub and the generator (Fig. 8), the front and rear bearing forces in 3 directions (Fig. 9).
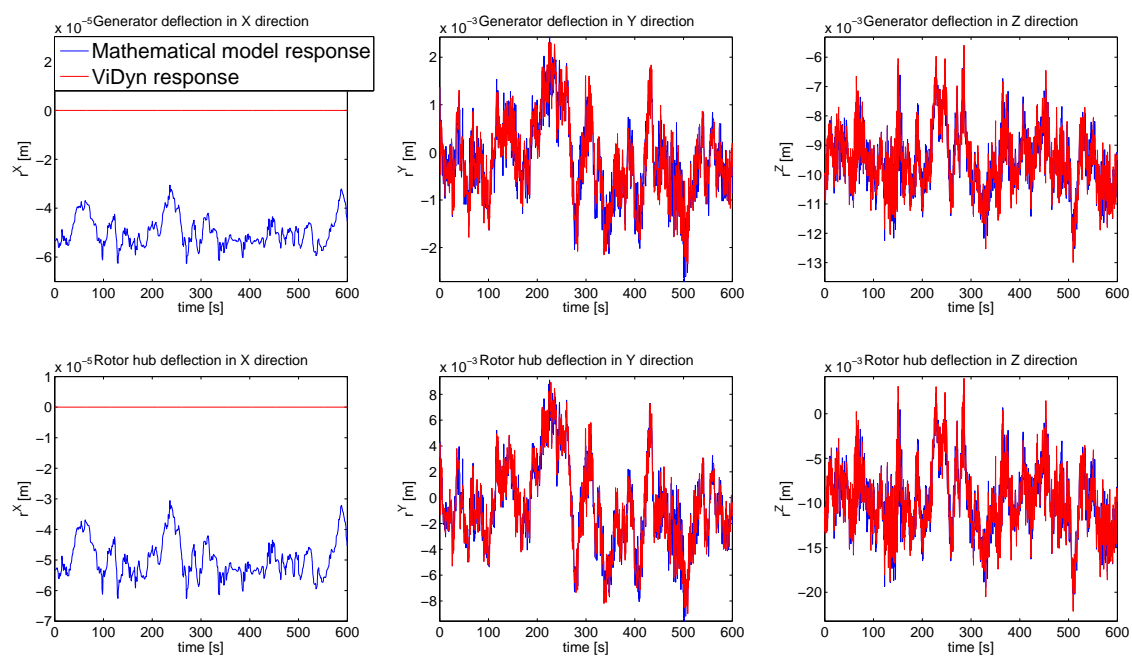

Fig. 8 The deflection fields at generator (up) and rotor hub (down), in $O S^{2}(11 \mathrm{~m} / \mathrm{s})$, Math - , and ViDyn models
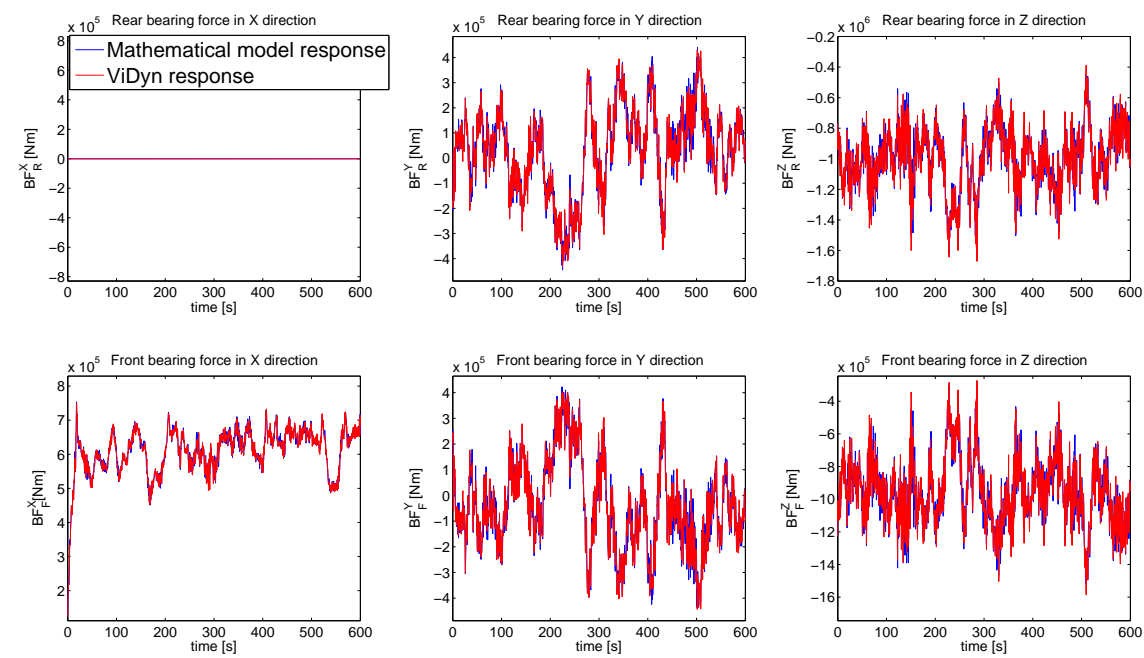

Fig. 9 The bearing forces in rear (up) and front (down), in $O S^{2}(11 \mathrm{~m} / \mathrm{s})$, Math , and ViDyn models 
- Normal operation at mean wind speed $18 \mathrm{~m} / \mathrm{s}\left(O S^{3}\right)$ :

For the operational scenario $O S^{3}$, the system response in both the mathematical model and the ViDyn simulation model is illustrated in terms of deflections at the hub and the generator (Fig. 10), the front and rear bearing forces in 3 directions (Fig. 11).
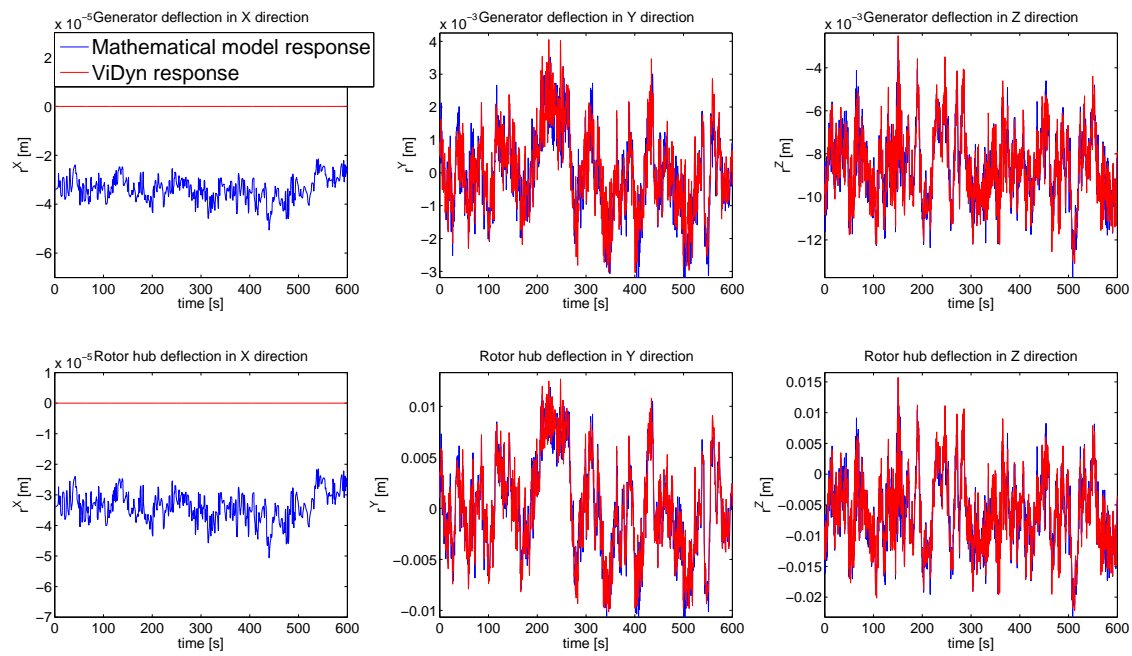

Fig. 10 The deflection fields at generator (up) and rotor hub (down), in $O S^{3}(18 \mathrm{~m} / \mathrm{s})$, Math , and ViDyn models
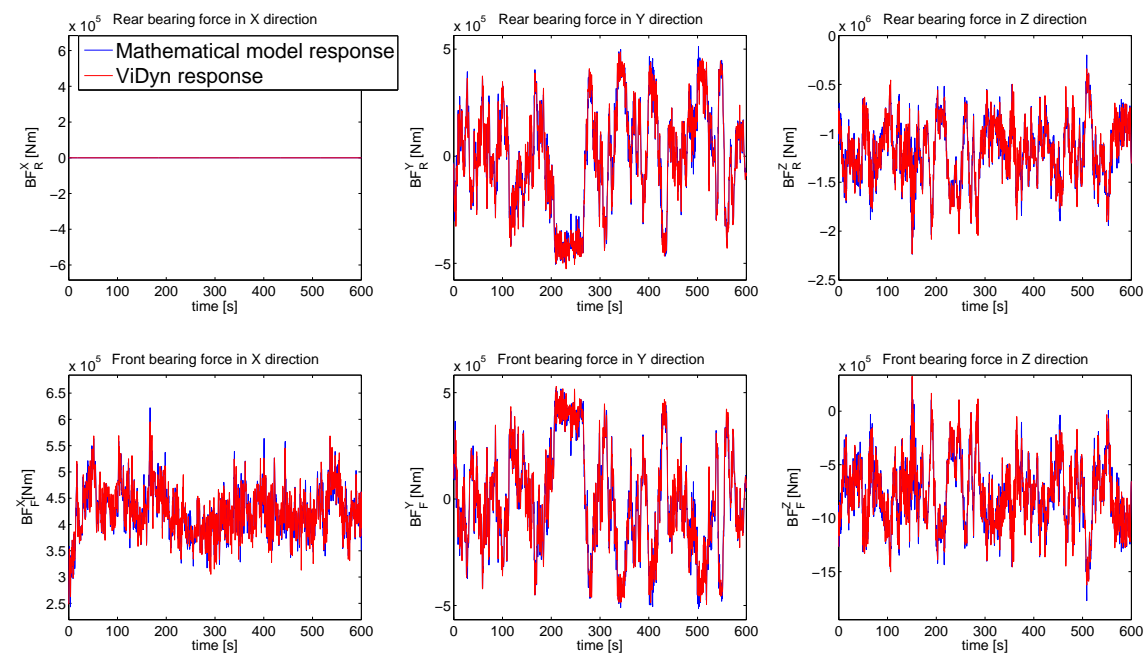

Fig. 11 The rear (left) and front (right) bearing forces, in $O S^{3}(18 \mathrm{~m} / \mathrm{s})$, Math , and ViDyn models 
Comparing the time domain results of the developed mathematical model and the ViDyn simulation, the two models were observed to correspond substantially. Thus, the mathematical simulation model is capable of capturing the dynamic response of the system to an acceptable level of accuracy in different operational scenarios. It should be noted that the mathematical model includes the hub and the generator deflections in $X$ direction, while in Vidyn this output of the system is not measured.

\subsubsection{Objective functions}

In order to survey a large number of different operations, objective functions can be defined in order to numerically quantify the system response. Depending on the specific application in mind, different objectives can be constructed. In this study, the following four objective functions are chosen:

- $\left[O F^{1}, O F^{2}\right]$

RMS values of tip deflection at the hub and at the generator:

The tip deflection is assessed in order to evaluate and quantify the motion of the hub and the generator. To this end, the deflection filed for the quasi-static solution with constant wind speed in all time domains is gathered $(\bullet s t)$, and is subtracted from the original deflection field for each specific wind speed. The definition of the ultimate deflection field is presented as follows:

$$
R=\sqrt{\left(X-X_{s t}\right)^{2}+\left(Y-Y_{s t}\right)^{2}+\left(Z-Z_{s t}\right)^{2}}
$$

where $X_{s t}, Y_{s t}$, and $Z_{s t}$ are the quasi-static equilibrium solutions for each specific mean wind speed. The static solution shows that for all cases there is a small increase in $Z_{s t}$ while increasing the wind speed. $X_{s t}$ and $Y_{s t}$ show no changes with wind speed.

- $\left[O F^{3}, O F^{4}\right]$

Bearing fatigue life index corresponding to the loads in the front and rear bearings:

The fatigue criteria used are based on the Palmgren-Miller rule, where the bearing forces lead to the damage index corresponding to the bearing fatigue estimation. The equivalent fatigue load $P$ is calculated based on the contribution of the radial forces and axial forces:

$$
P=A_{1} F_{r}+A_{2} F_{X}=A_{1} \sqrt{F_{B}^{Y^{2}}+F_{B}^{Z^{2}}}+A_{2} F_{B}^{X}
$$

where $A_{1}=2.5$ and $A_{2}=3.7$ are chosen for spherical roller bearings [29]. Calculating a time-history of bearing forces from the mathematical model, the damage index $D I$ rate is computed using the Palmgren-Miner rule as:

$$
\begin{aligned}
& D I=\frac{1}{T_{\text {sim }}} \sum_{i=1}^{N_{\text {rev }}} \frac{1}{L_{i}}, \\
& L_{i}=\underbrace{a_{1} a_{2}}_{1} a_{3}\left(P_{i}\right)\left(\frac{C}{P_{i}}\right)^{P} .
\end{aligned}
$$

where $T_{\text {sim }}$ is the simulation time (600 sec for $O S^{1-3}$ ), $N_{\text {rev }}$ is the number of revolutions during the simulation, $L_{i}$ is the $L_{10}$ - life associated with an equivalent load $P$ (Eq. (29)) obtained from the bearing-specific combination of axial and radial forces where $A_{1}, A_{2}, a_{1} a_{2} C, P$ are parameters, and $a_{3}(\bullet)$ is a function specified by the bearing design [30].

Of course, this is a rather crude measure of bearing damage and excludes several other mechanisms leading to failures in bearings [31]. 
The following figure illustrates the response of both mathematical and ViDyn models in terms of equivalent radial deflection and equivalent bearing force, defined in Eq. (28) and Eq. (29), respectively.
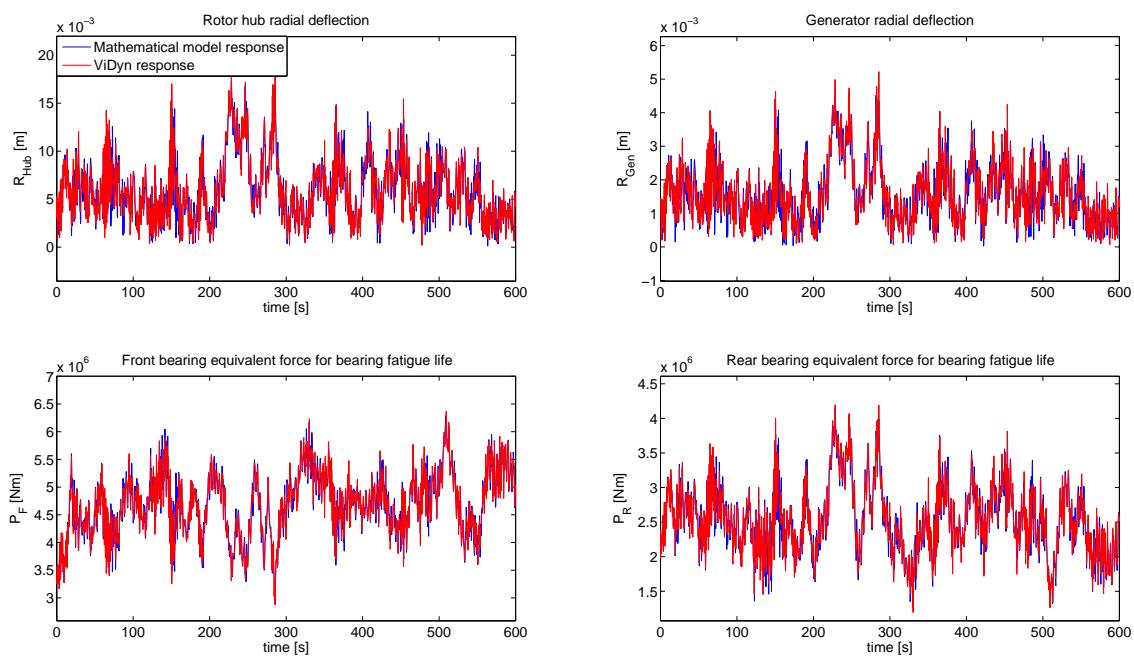

Fig. 12 The equivalent radius (up) in the hub (left) and the generator (right) locations, and the equivalent bearing forces (below) in front (left) and rear bearings (right), in a normal operational scenario at mean wind speed $O S^{2}(11 \mathrm{~m} / \mathrm{s})$, Math — ViDyn - models

To estimate the agreements between mathematical (full and simplified) model with the ViDyn model, the time domain values presented in Fig. 12, namely the equivalent radial deflections at the hub and the generator, as well as the front and rear bearing forces, have been used to calculate the radial deflections (Fig. 13), and the bearings damage indices DI (Fig. 14), respectively. 
The comparison between the full and simplified models versus the ViDyn model has been illustrated in terms of the computed objective functions in Figs. 13- 14.
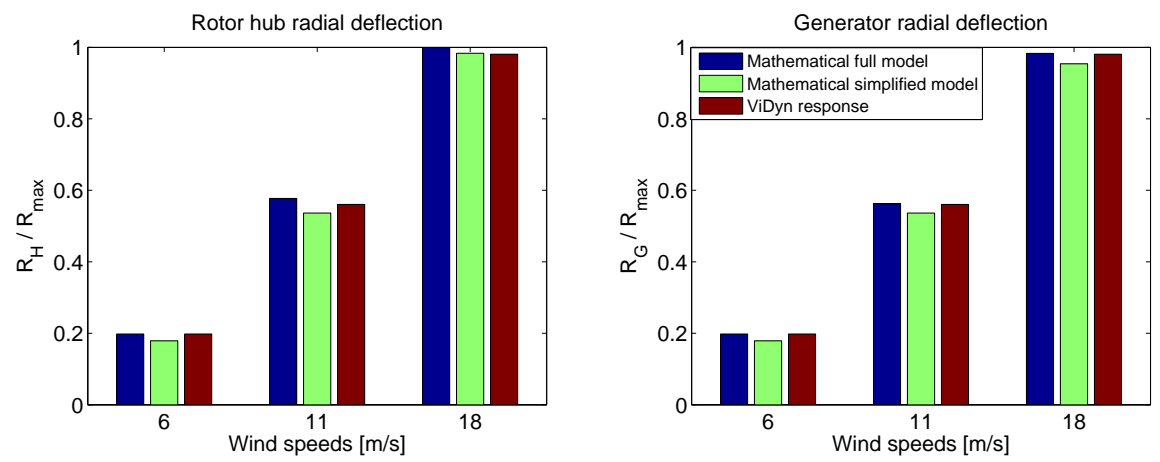

Fig. 13 The scaled radial deflection in the rotor hub (left) and the generator (right), for full

) and simplified ( $\longrightarrow$ ) mathematical models, and ViDyn model ( -) in all operational scenarios $\left(O S^{1-3}\right), R_{\max }$ is the maximum value of the radial deflection encountered in all studied scenarios.
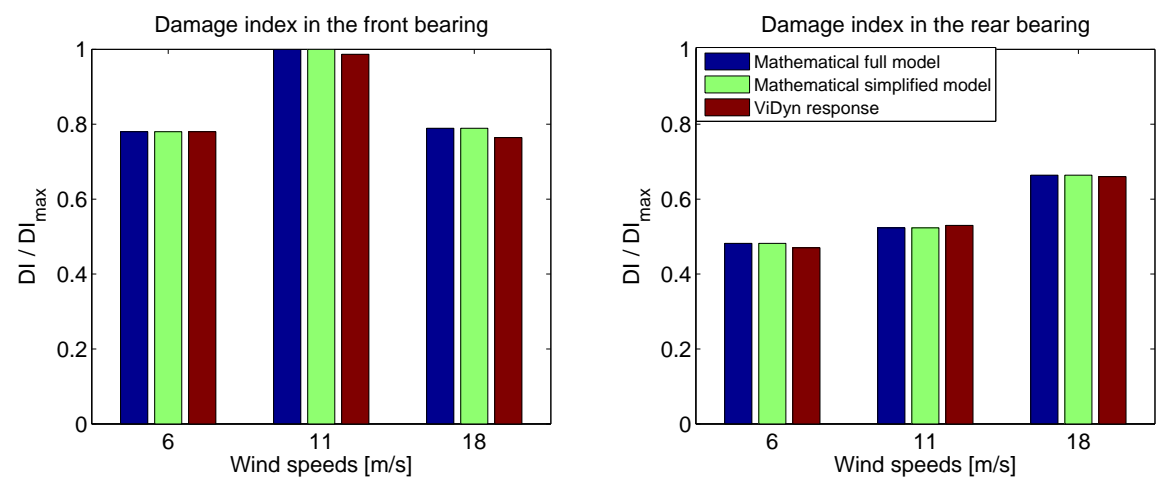

Fig. 14 The scaled damage index in the front (left) and rear (right) bearings, for full ( ) and simplified ( -) mathematical models, and ViDyn model (__ in all operational scenarios $\left(O S^{1-3}\right), D I_{\max }$ is the maximum value of bearing damage encountered in all studied scenarios. 
As seen the mathematical response prediction, in terms of shaft deflection at the generator and the hub, as well as damage index in bearings, there is quite good agreement with the ViDyn model. The damage indices in both the full and simplified mathematical models are also quite similar, in particular with the bearing forces, which is understandable since the flexibility of the main shaft does not directly affect the bearing forces.

As demonstrated in Figs. 13- 14, the tip deflection differences in both the full and simplified models are insignificant compared to the minimum radius in the ViDyn model $([0.6,1.6,2.5] \mathrm{mm})$ at 3 different wind speeds. The tip deflection in the vertical direction is larger than in the lateral deflection, and thus dominates the output function.

\section{System response under different wind conditions}

In this section, the system response quantified by the objective functions is studied under different wind conditions, where the effect of wind speed, turbulence intensity and incoming wind vertical inclination is reported. For each specific wind speed, $N_{\text {real }}=20$ wind realization samples are studied. The wind fields for a specific wind speed are obtained from random realizations based on the Kaimal spectra as described in the standard IEC-61400 [33] characterized by turbulence intensity factor $I_{r e f}$. The following cases are studied:

- Turbulence intensity factor $I_{\text {ref }}=[0.05,0.12,0.20]$ for wind speeds $[5,6, \ldots, 20] \mathrm{m} / \mathrm{s}$, each for $N_{\text {real }}(=20)$ different wind realization, The results are reported in Fig. 16 - 18.

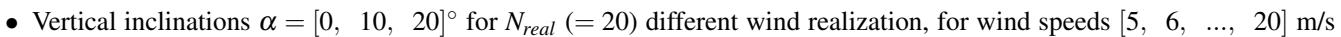
and different turbulence intensity factor. The results are shown in Fig. 19 - 22.

The turbine system model has been adjusted to avoid cut-out due to high wind or large yaw forces which otherwise occur at the higher wind speeds. Note that the damage and deflection functions in Fig. 16- 22 are scaled with respect to $D I_{\text {max }}$ (maximum $D I$ value encountered in the studied scenarios), and $R_{\max }$ (maximum $R$ value encountered in the studied scenarios). 


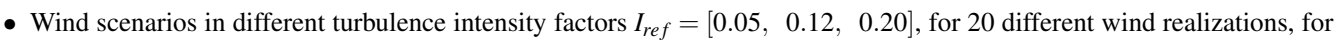
wind speeds $[5,6, \ldots, 20] \mathrm{m} / \mathrm{s}$.
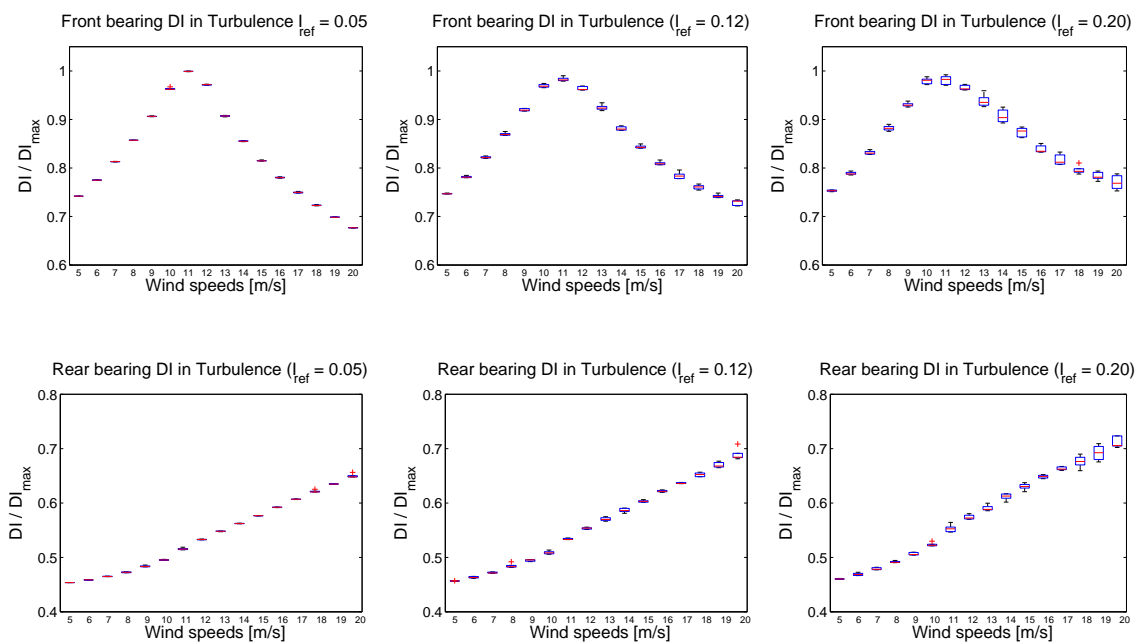

Fig. 15 The scaled damage index DI in the mathematical model in front (above) and rear (below) bearings for the turbulence intensity factors 1 (left), 2 (middle), and 3 (right)
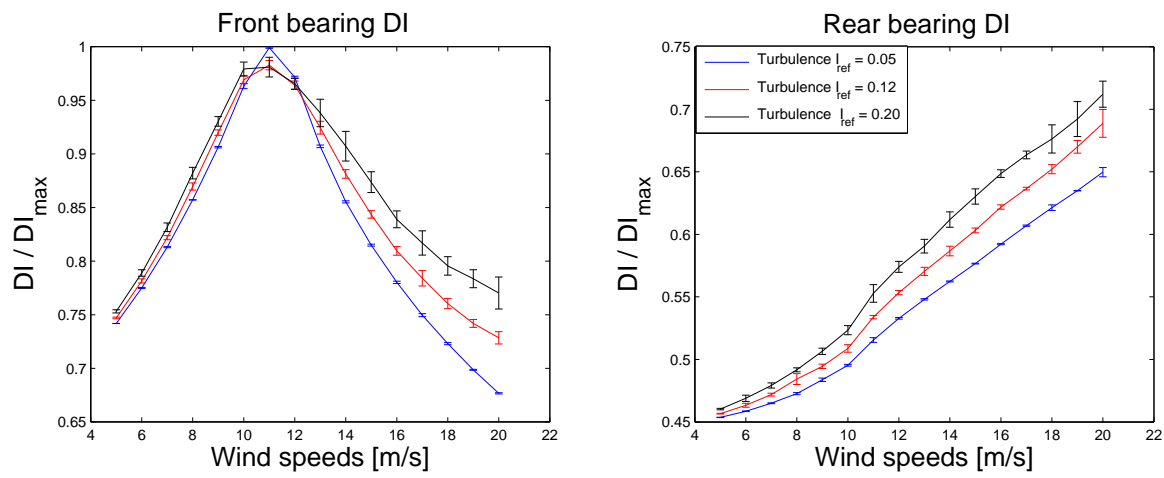

Fig. 16 The scaled damage index $D I$ in the mathematical model in front (left) and rear (right) bearings, for different turbulence intensity factors $I_{r e f}=\left[\begin{array}{lll}0.05, & 0.12, & 0.20\end{array}\right]$ 
For the tip deflection radius in the hub and the generator the same set of simulations has been performed and the results are shown below (Fig. 17- 18):
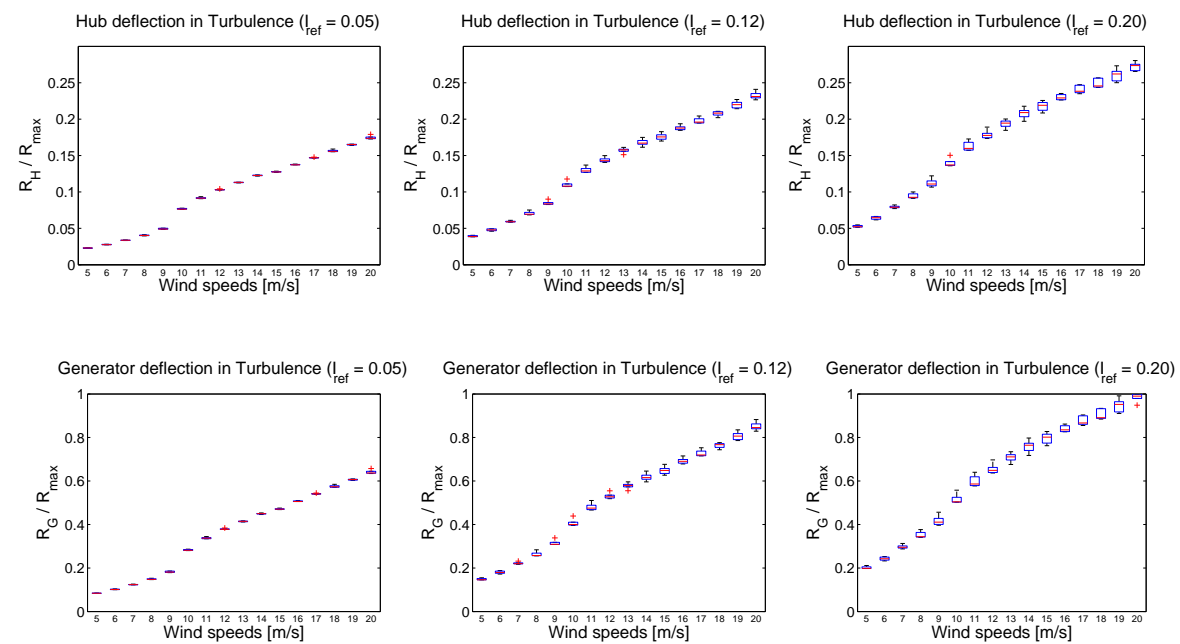

Fig. 17 The scaled tip deflection radius $R$ in the mathematical model in the hub (above) and the generator (below), for the turbulence intensity factors 1 (left), 2 (middle), and 3 (right)
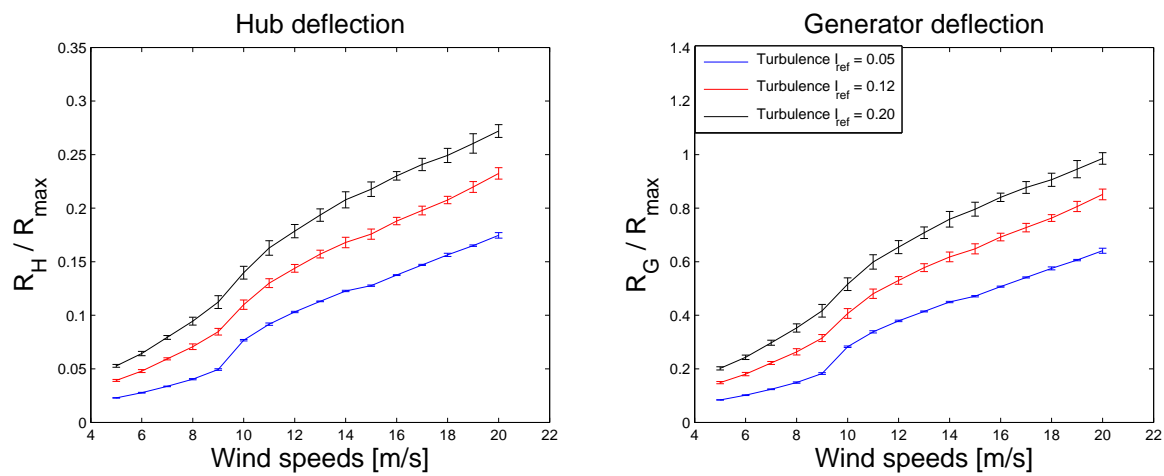

Fig. 18 The scaled tip deflection radius $R$ in the mathematical model in the hub (left) and the generator (right), for different turbulence intensity factors $I_{\text {ref }}=\left[\begin{array}{ll}0.05,0.12,0.20\end{array}\right]$ 


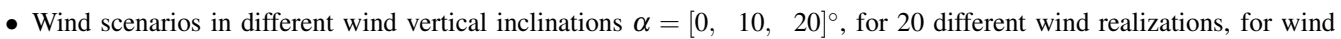
speeds $[5,6, \ldots, 20] \mathrm{m} / \mathrm{s}$ and different turbulence intensity factors.
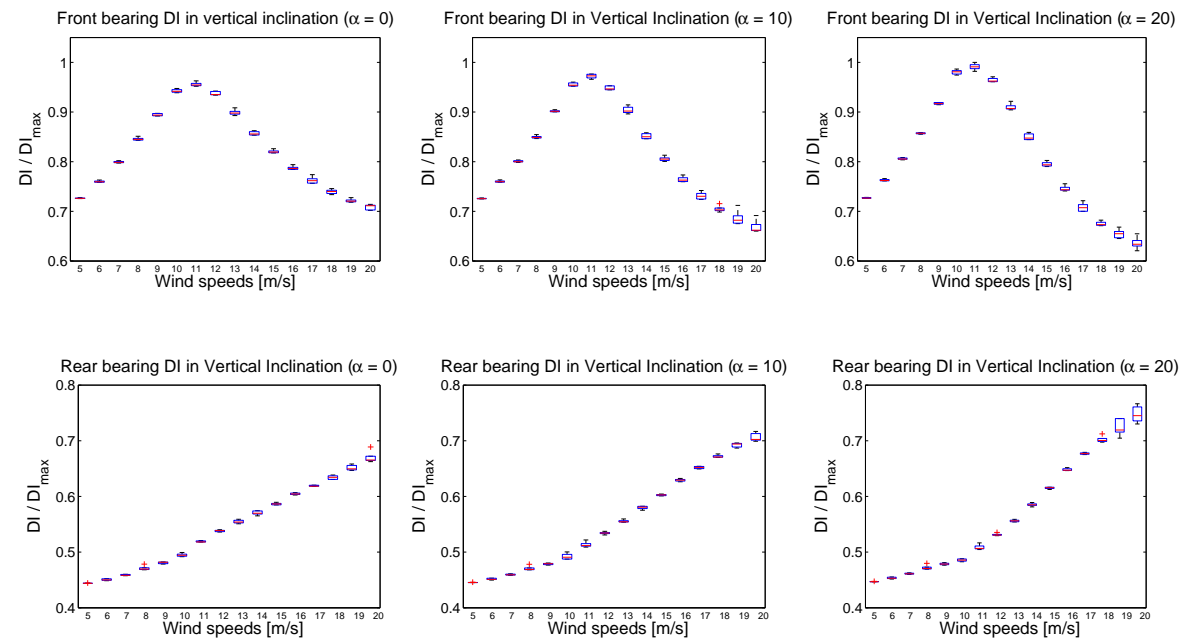

Fig. 19 The scaled damage index DI in the mathematical model in front (above) and rear (below) bearings for the vertical inclination angles $0^{\circ}$ (left), $10^{\circ}$ (middle), and $20^{\circ}$ (right)
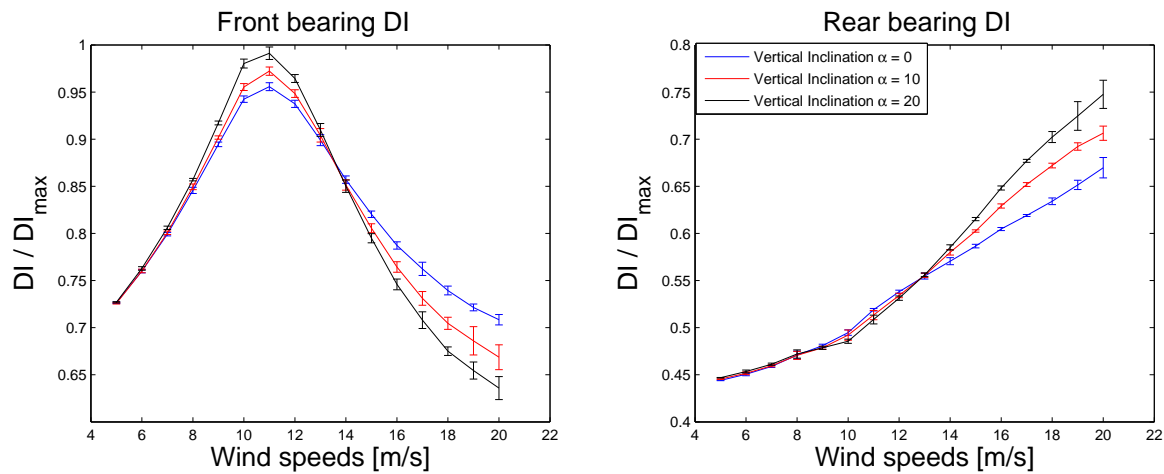

Fig. 20 The scaled damage index $D I$ in the mathematical model in front (left) and rear (right) bearings, for different vertical inclination angles $[0,10,20]^{\circ}$ 

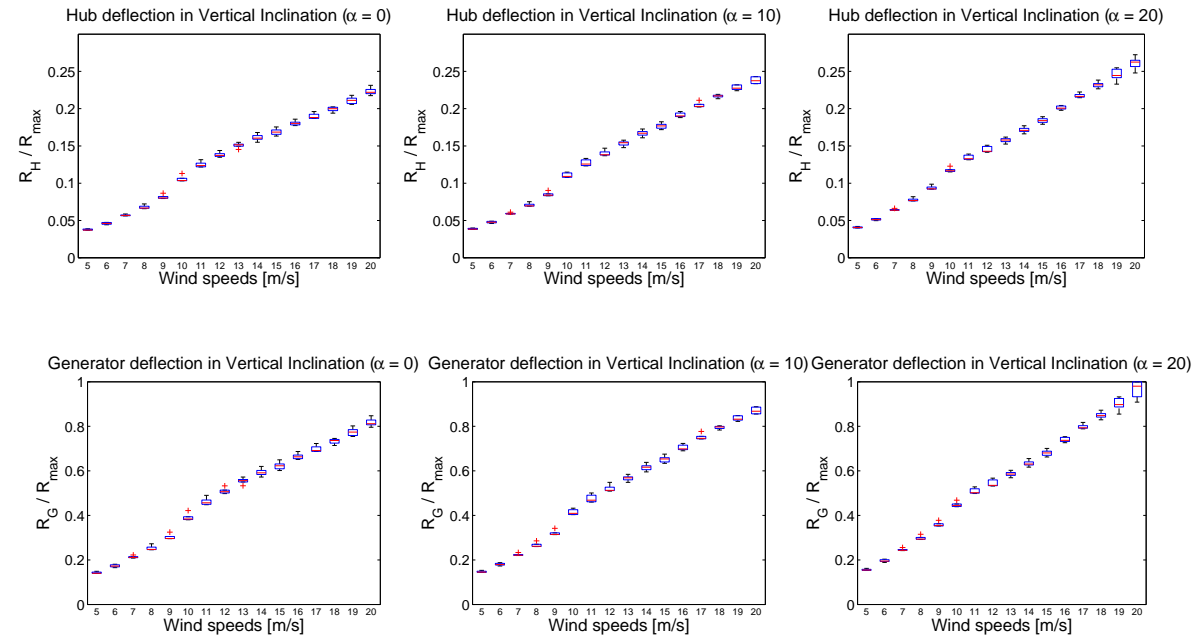

Fig. 21 The scaled tip deflection $R$ in the mathematical model in the hub (above) and the generator (below), for the vertical inclination angles $0^{\circ}$ (left), $10^{\circ}$ (middle), and $20^{\circ}$ (right)
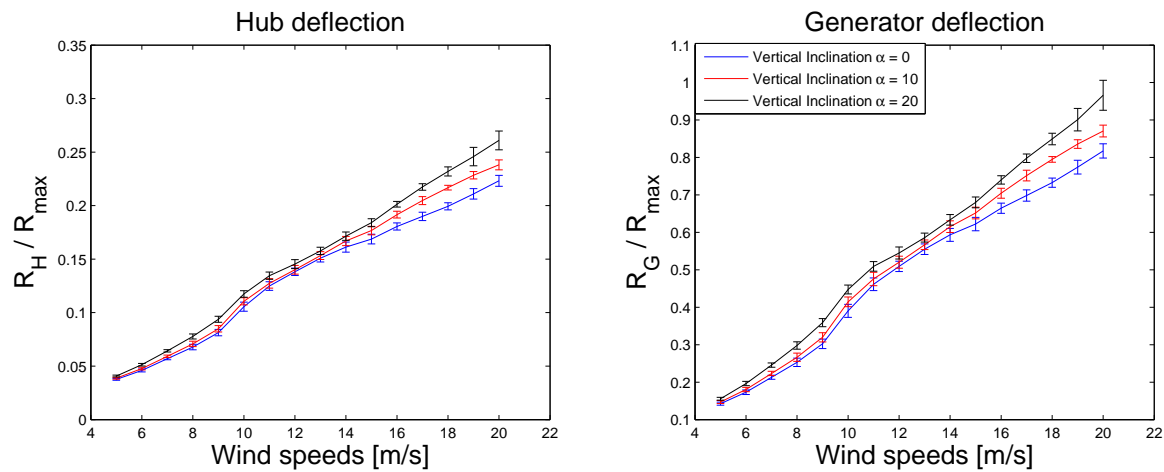

Fig. 22 The scaled tip deflection radius $R$ in the mathematical model in the hub (left) and the generator (right), for different vertical inclination angles $[0,10,20]^{\circ}$ 
As can be seen in Figs 15 and 20, the largest damage index rate for the front bearing occurs at $11 \mathrm{~m} / \mathrm{s}$ in all operational scenarios, which is the region where rotor blade pitch control starts. The pitch control reduces the axial thrust load, and consequently, the equivalent load $P$. For the rear bearing, the mean damage index rate and variability increase accordingly with increased mean wind speed. It has been seen that for each wind speed, both a higher turbulence intensity factor and vertical inclination angle cause more damage in both bearings. However, in Fig. 20, the damage rate in the front main bearing above 11 $\mathrm{m} / \mathrm{s}$ is decreased by increasing vertical inclination. The radial deflections at the hub and the generator increase for higher wind speeds, when the turbulence intensity factor or the vertical inclination angle is increased (Figs. 18, 22). This is in some ways to be expected, since with higher turbulence factor and vertical inclination angle, the wind turbine experiences more extreme loads, and ultimately higher deflection ranges at the hub and generator. In higher turbulence cases as well as inclination angles, the range of measured outputs is increased, especially for higher wind speeds, which appears reasonable as the turbulence and effect of inclination scales with wind speed. It is noted that Figs. 15, 17, 19, 21, also illustrate the standard deviation for each scenario separately.

\section{Conclusions and outlook}

In this paper, a mathematical model for a direct drive train wind turbine was developed. The intended use of the developed model is to evaluate drive train performance from measured blade root forces and moments. The model was shown to agree well with the turbine system simulation tool ViDyn in terms of bearing forces and deflection at main shaft ends. The developed mathematical model was further used to quantify drive train performance under varying wind speed, turbulence and vertical inclination of incoming wind. This quantification was performed in terms of objective functions measuring a representative value of shaft deflection at hub and generator, as well as bearing forces (in terms of fatigue index). The results are in agreement with other studies and give further confidence in the model.

From the present study of a direct drive wind turbine, the following was observed:

- The predicted bearing forces were almost identical considering the main shaft as either a rigid or a flexible body, concluding that shaft flexibility has little effect on bearing fatigue.

- The objective functions displayed an increase in variation between wind speed realizations for increased wind speed, meaning that there is a much larger degree of uncertainty in predicted drive train behavior at higher wind speeds.

- The shaft axial flexibility was shown to have a negligible effect on the drive train performance, and can be skipped (which, due to its high frequency, saves substantial computational time)

Proposals for further studies include

- A study of extreme and transient events, such as start-up, shut-down, grid faults, etc. However, such a study would require a more careful model of the generator, in particular separating the generator stator and rotor into separate bodies with inertia.

- Additional cases with respect to wind parameters such as turbulence characteristics should be explored, as well as other conditions (start-up and shut-downs, extreme gust with direction change, ice on blades, etc.). One example could be the scenario with wakes in the wind field that may appear randomly in the swept area and how these affect the objective functions.

- Apply global sensitivity analysis to the developed multibody dynamic model, in order to assess the sensitivity of input structural and excitation parameters to the objective functions. For instance, since many of the structural parameters come with a large level of uncertainty (e.g. bearing stiffness), it is of interest to see how this uncertainty carries over to objective functions.

- Considering the front main bearing, it seems that wind speeds around the start of pitching require most attention, whereas for the rear main bearing it is not as obvious, as high wind speeds are significantly less frequent.

- If to adopt the present approach to indirect drive turbine, additional objectives should be studied, in particular with respect to gearbox life. 


\section{ACKNOWLEDGEMENTS}

This project is financed through the Swedish Wind Power Technology Center (SWPTC). SWPTC is a research center for the design of wind turbines. The purpose of the center is to support Swedish industry with knowledge of design techniques as well as maintenance in the field of wind power. The Centre is funded by the Swedish Energy Agency and Chalmers University of Technology together with academic and industrial partners.

\section{Appendix}

The following section contains the detailed derivation of governing equation within the developed mathematical model of the direct drive wind turbine based on floating reference frame. The section includes the mass matrix derivation and arguments (sec. 6.1), derivation of elastic forces (sec. 6.2), the damping coefficients (sec. 6.3), the bearing forces (sec. 6.4), the wind loads (sec. 6.5), the generator loads (sec. 6.6), derivation of the quadratic velocity vector (sec. 6.7).

\subsection{Inertia of deformable bodies}

This section contains a detailed formulation of the mass matrix and quadratic velocity vector. The mass matrix $\mathbf{M}$ can be written in a partitioned form as

$$
\mathbf{M}=\left[\begin{array}{ccc}
\mathbf{m}_{R R} & \mathbf{m}_{R \theta} & \mathbf{m}_{R f} \\
\mathbf{S y m}_{\theta \theta} & \mathbf{m}_{\theta f} \\
& \mathbf{m}_{f f}
\end{array}\right]
$$

where the mass matrix arguments are defined as followed:

$$
\begin{aligned}
& \mathbf{m}_{R R}^{i}=\int_{V^{i}} \rho^{i} \mathbf{I} d V^{i}=\left[\begin{array}{ccc}
m^{i} & 0 & 0 \\
0 & m^{i} & 0 \\
0 & 0 & m^{i}
\end{array}\right]=m \mathbf{I} \approx \sum_{j=1}^{n_{j}} m^{i j} \mathbf{I}, \\
& \mathbf{m}_{R \theta}^{i}=\int_{V^{i}} \rho^{i} \mathbf{B}^{i} d V^{i}=-\int_{V^{i}} \rho^{i} \mathbf{A}^{i} \tilde{\tilde{\mathbf{u}}}^{i} \overline{\mathbf{G}}^{i} d V^{i}=-\mathbf{A}^{i} \underbrace{\left[\int_{V^{i}} \rho^{i} \tilde{\tilde{\mathbf{u}}}^{i} d V^{i}\right]}_{\tilde{\mathbf{S}}^{i}} \overline{\mathbf{G}}^{i} \approx \sum_{j=1}^{n_{j}} m^{i j} \mathbf{B}^{i j T}, \\
& \mathbf{m}_{R f}^{i}=\mathbf{A}^{i} \int_{V^{i}} \rho^{i} \mathbf{S}^{i} d V^{i} \approx \sum_{j=1}^{n_{j}} m^{i j} \mathbf{I} \approx \sum_{j=1}^{n_{j}} m^{i j} \mathbf{N}^{i j T} \mathbf{A}^{i T}, \\
& \mathbf{m}_{\theta \theta}^{i}=\int_{V^{i}} \rho^{i} \overline{\mathbf{G}}^{i T} \tilde{\widetilde{\mathbf{u}}}^{i T} \tilde{\widetilde{\mathbf{u}}}^{i} \overline{\mathbf{G}}^{i} d V^{i}=\overline{\mathbf{G}}^{i} \underbrace{\left[\int_{V^{i}} \rho^{i} \tilde{\tilde{\mathbf{u}}}^{i T} \tilde{\mathbf{u}}^{i} d V^{i}\right]}_{\tilde{\mathbf{I}}_{\theta \theta}^{i}} \overline{\mathbf{G}}^{i} \approx \sum_{j=1}^{n_{j}} m^{i j} \mathbf{B}^{i j T} \mathbf{B}^{i j},
\end{aligned}
$$

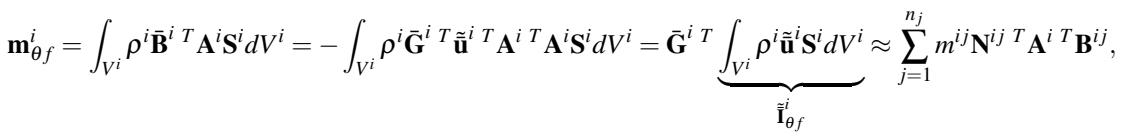

$$
\begin{aligned}
& \mathbf{m}_{f f}^{i}=\int_{V^{i}} \rho^{i} \mathbf{S}^{i T} \mathbf{S}^{i} d V^{i}=\mathbf{S}_{11}^{i}+\mathbf{S}_{22}^{i}+\mathbf{S}_{33}^{i} \approx \sum_{j=1}^{n_{j}} m^{i j} \mathbf{N}^{i j T} \mathbf{N}^{i j} .
\end{aligned}
$$

Here, $i$ denotes the bodies in the system structure containing the main shaft, generator and hub at both ends.

Note that $\tilde{\bullet}$ is skew symmetric matrix $(\tilde{\bullet}=\bullet-\bullet T)$.

The definition of the A matrix:

$$
\mathbf{A}^{T}=\mathbf{R}_{X}(\phi) \mathbf{R}_{Y}(\theta) \mathbf{R}_{Z}(\psi)
$$

where $\mathbf{R}_{i}$ s are rotational matrices around different axes.

where the three angles $\phi, \theta$, and $\psi$ are the Euler angles. The matrix $\mathbf{A}$ in Eq. 33 is the transformation matrix expressed in terms 
of Euler angles.

Moreover, $\mathbf{B}^{i}=\mathbf{B}^{i}\left(\theta^{i}, \mathbf{q}_{f}^{i}\right)$ is defined as:

$$
\mathbf{B}^{i}=\left[\frac{\partial}{\partial \theta_{k}^{i}}\left(\mathbf{A}^{i} \overline{\mathbf{u}}^{i}\right)\right]=-\mathbf{A}^{i} \tilde{\mathbf{u}}^{i} \overline{\mathbf{G}}^{i}
$$

where $k=1, \ldots, n_{k} . n_{k}$ is total number of rotational coordinates of the reference of body $i$.

Following up $m_{\theta \theta}$ derivation, $\tilde{\overline{\mathbf{I}}}_{\theta \theta}^{i}$ represents the inertia tensor of the deformable body $i$ and is defined as follows:

$$
\tilde{\tilde{\mathbf{I}}}_{\theta \theta}^{i}=\int_{V^{i}} \rho^{i} \tilde{\mathbf{u}}^{i T} \tilde{\mathbf{u}}^{i} d V^{i}
$$

Noting that $\tilde{\mathbf{u}}^{i T}=-\tilde{\tilde{\mathbf{u}}}$ the following holds for $\tilde{\overline{\mathbf{I}}}_{\theta \theta}^{i}$ :

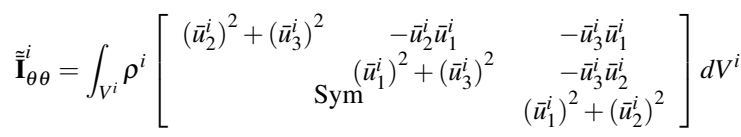

Following up $m_{\theta f}$ derivation, $\tilde{\overline{\mathbf{I}}}_{\theta f}^{i}$ could be defined as follows:

$$
\tilde{\overline{\mathbf{I}}}_{\theta f}^{i}=\int_{V^{i}} \rho^{i}\left[\begin{array}{c}
\mathbf{q}_{f}^{i}\left(\mathbf{S}_{2}^{i T} \mathbf{S}_{3}^{i}-\mathbf{S}_{3}^{i T} \mathbf{S}_{2}^{i}\right) \\
\mathbf{q}_{f}^{i T}\left(\mathbf{S}_{3}^{i T} \mathbf{S}_{1}^{i}-\mathbf{S}_{1}^{i T} \mathbf{S}_{3}^{i}\right) \\
\mathbf{q}_{f}^{i}{ }^{T}\left(\mathbf{S}_{1}^{i}{ }^{T} \mathbf{S}_{2}^{i}-\mathbf{S}_{2}^{i T} \mathbf{S}_{1}^{i}\right)
\end{array}\right] d V^{i}+\int_{V^{i}} \rho^{i}\left[\begin{array}{c}
\left(x_{2}^{i} \mathbf{S}_{3}^{i}-x_{3}^{i} \mathbf{S}_{2}^{i}\right) \\
\left(x_{3}^{i} \mathbf{S}_{1}^{i}-x_{1}^{i} \mathbf{S}_{3}^{i}\right) \\
\left(x_{1}^{i} \mathbf{S}_{2}^{i}-x_{2}^{i} \mathbf{S}_{1}^{i}\right)
\end{array}\right] d V^{i}
$$

Note that $\mathbf{G}$ and $\mathbf{A}$ are not space-dependent and thus could be extracted from the integral. The definition for transformation matrix $\mathbf{A}$ is based on Euler angles which are widely used and it is a rotation of an angle $\phi$ about the $\mathbf{X}_{1}^{i}$ axis, followed $\mathbf{b}$ rotation $\theta$ about the $\mathbf{X}_{2}^{i}$ axis, followed b rotation $\psi$ about the $\mathbf{X}_{3}^{i}$ axis. Consequently, the definition for the $\overline{\mathbf{G}}$ matrix, which is dependent of the generalized rotational parameters would be as follows:

$$
\overline{\mathbf{G}}=\left[\begin{array}{ccc}
\cos \theta \cos \psi & \sin \psi & 0 \\
-\cos \theta \sin \psi & \cos \psi & 0 \\
\sin \theta & 0 & 1
\end{array}\right]
$$

\subsection{Elastic forces components}

The stiffness matrix associated with the elastic coordinates of the main shaft is presented as follows:

$$
\mathbf{K}_{f f}^{m s}=\int_{0}^{l}(\mathbf{D S})^{T} \mathbf{E} \mathbf{D S} d x
$$

where the stiffness matrix is defined as follows:

$$
\mathbf{E}=\left[\begin{array}{cccc}
E A & 0 & 0 & 0 \\
0 & G J & 0 & 0 \\
0 & 0 & E I_{y} & 0 \\
0 & 0 & 0 & E I_{z}
\end{array}\right]
$$

The DS matrix represents the higher derivations of the shape function $\mathbf{S}$ defined in Eq. (22) and is:

$$
\mathbf{D S}(x)=\left[\begin{array}{cccccc}
\left(m_{X}^{1}(x)\right)^{\prime} & 0 & 0 & 0 & 0 & 0 \\
0 & \left(m_{T}^{1}(x)\right)^{\prime} & 0 & 0 & 0 & 0 \\
0 & 0 & \left(m_{B}^{1}(x)\right)^{\prime \prime} & \left(m_{B}^{2}(x)\right)^{\prime \prime} & 0 & 0 \\
0 & 0 & 0 & 0 & \left(m_{B}^{1}(x)\right)^{\prime \prime} & \left(m_{B}^{2}(x)\right)^{\prime \prime}
\end{array}\right]
$$


The flexible parts of the mass matrix $\mathbf{M}_{f f}$, the stiffness matrix $\mathbf{K}_{f f}$, and the damping matrix $\mathbf{C}_{f f}$ components could be defined as follows:

$$
\begin{aligned}
& \mathbf{M}_{f f}=\operatorname{diag}\left(M_{f f}^{x}, M_{f f}^{t}, \mathbf{M}_{f f}^{b}\right), \\
& \mathbf{K}_{f f}=\operatorname{diag}\left(K_{f f}^{x}, K_{f f}^{t}, \mathbf{K}_{f f}^{b}\right), \\
& \mathbf{C}_{f f}=\operatorname{diag}\left(C_{x}, C_{t}, \mathbf{C}_{b}\right) .
\end{aligned}
$$

The arguments of the damping matrix $\mathbf{C}_{f f}$ are derived and defined in Eq.(43a)-(44b).

\subsection{Damping coefficients (Rayleigh damping factor)}

Upon imposing the Rayleigh damping factor [25,26], the corresponding damping coefficient for the flexibility DOF is computed as follows:

$$
\begin{aligned}
& \mathbf{C}_{b}=\eta \mathbf{M}_{f f}^{b}+\delta \mathbf{K}_{f f}^{b}, \\
& C_{x}=2 \zeta \sqrt{K_{f f}^{x} M_{f f}^{x}}, \\
& C_{t}=2 \zeta \sqrt{K_{f f}^{t} M_{f f}^{t}} .
\end{aligned}
$$

where $\mathbf{C}_{b}, C_{x}$ and $C_{t}$ are the contribution of bending, elongation and torsional modes within the flexible DOF into the system damping. Note that $\delta$, and $\eta$ are the Rayleigh damping coefficients, and are defined as follows:

$$
\begin{aligned}
& \delta=2 \zeta\left(\omega_{1}+\omega_{2}\right), \\
& \eta=2 \zeta \frac{\omega_{1} \omega_{2}}{\left(\omega_{1}+\omega_{2}\right)} .
\end{aligned}
$$

where $\omega_{i}$ is the eigenfrequencies of the system.

Finally, for $\mathbf{C}_{f f}$ the following relation holds

$$
\mathbf{C}_{f f}=\operatorname{diag}\left(C_{x}, C_{t}, \mathbf{C}_{b}\right)
$$

\subsection{Bearing forces}

This section addresses the forces appearing in the bearings mounted along the main shaft, attaching the main shaft ( $\bullet$ ms) to the bedplate $\left(\bullet_{b p}\right)$. In this section, the derivation of bearing forces is presented. First, the front and rear bearing locations with respect to the bedplate are given in terms of $\mathbf{q}_{m s}$ as follows:

$$
\begin{aligned}
& \mathbf{r}_{b p}^{F}=\mathbf{R}_{b p}+\mathbf{A}\left(\theta_{b p}\right) \overline{\mathbf{u}}_{b p}^{F} \\
& \mathbf{r}_{b p}^{R}=\mathbf{R}_{b p}+\mathbf{A}\left(\theta_{b p}\right) \overline{\mathbf{u}}_{b p}^{R} .
\end{aligned}
$$

where the locations of the front and rear bearings in the bedplate coordinate system are $\overline{\mathbf{u}}_{b p}^{F}=\left[-L_{m}, 0, h_{m s}\right]^{T}$ and $\overline{\mathbf{u}}_{b p}^{R}=\left[L_{m}, 0, h_{m s}\right]^{T}$, respectively. Also, $L_{m}=\frac{1}{2}\left(L_{1}+L_{2}\right)$ is the middle point of the bedplate (position of yaw bearing). Correspondingly, the bearing location with respect to the main shaft is:

$$
\begin{aligned}
& \mathbf{r}_{m s}^{F}=\mathbf{R}_{m s}+\mathbf{A}\left(\theta_{m s}\right) \overline{\mathbf{u}}_{m s}^{F}\left(L_{1}\right), \\
& \mathbf{r}_{m s}^{R}=\mathbf{R}_{m s}+\mathbf{A}\left(\theta_{m s}\right) \overline{\mathbf{u}}_{m s}^{R}\left(L_{2}\right) .
\end{aligned}
$$

In case $\mathbf{S}\left(L_{1}\right)=0$, the expression $\overline{\mathbf{u}}_{m s}^{F}=\left[L_{1}, 0,0\right]^{T}$ holds. The relative position of an attached point in main shaft with respect to the attached point in the bedplate is expressed in Eq. (15).

By defining the spring length between two bodies as a vector in 3 coordinates (here main shaft and bearing housing interconnected to the bedplate) and its time derivative, the force along the spring-damper element is defined as follows:

$$
\mathbf{F}=\mathbf{K}_{B H}^{i} \mathbf{l}+\mathbf{C}_{B H}^{i} \mathbf{i}
$$


where stiffness $\mathbf{K}_{B H}^{i}$ and damping $\mathbf{K}_{B H}^{i}$ are defined as follows:

$$
\begin{aligned}
& \mathbf{K}_{B H}^{i}=\operatorname{diag}\left(K_{B H_{X}}^{i}, K_{B H_{Y}}^{i}, K_{B H_{Z}}^{i}\right), \\
& \mathbf{C}_{B H}^{i}=\operatorname{diag}\left(C_{B H_{X}}^{i}, C_{B H_{Y}}^{i}, C_{B H_{Z}}^{i}\right) .
\end{aligned}
$$

and for the displacement vector we have:

$$
\mathbf{I}=\left[l_{X}, l_{Y}, l_{Z}\right]^{T}
$$

The generalized force vector for body $i$ contributing the bearing forces to the dynamics of the system could be defined as follows:

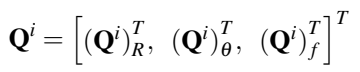

where the corresponding arguments are determined below:

$$
\begin{aligned}
& (\mathbf{Q})_{R}{ }^{T}=-\mathbf{F}^{T}, \\
& (\mathbf{Q})_{\theta}{ }^{T}=-\mathbf{F}^{T} \mathbf{B}^{i}, \\
& (\mathbf{Q})_{f}{ }^{T}=-\mathbf{F}^{T} \mathbf{A} \mathbf{S} .
\end{aligned}
$$

\subsection{Wind loads}

The more detailed derivation related to the sec. 2.6 .1 is presented here. The force and moment components of the wind loads are defined as follows:

$$
\begin{aligned}
\mathbf{Q}_{R}^{F} & =\mathbf{F}^{T}, \\
\mathbf{Q}_{\theta}^{F} & =\mathbf{F}^{T} \mathbf{B}, \\
\mathbf{Q}_{f}^{F} & =\mathbf{F}^{T} \mathbf{A} \mathbf{S}(0) .
\end{aligned}
$$

$$
\begin{aligned}
\mathbf{Q}_{R}^{M} & =0 \\
\mathbf{Q}_{\theta}^{M} & =\mathbf{T}^{T}+\mathbf{T}^{T} \mathbf{B}_{h u b}, \\
\mathbf{Q}_{f}^{M} & =\mathbf{T}^{T} \mathbf{A}\left[\begin{array}{cccccc}
0 & m_{T}^{1}(0) & 0 & 0 & 0 & 0 \\
0 & 0 & 0 & 0 & -\left(m_{B}^{1}(0)\right)^{\prime} & -\left(m_{B}^{2}(0)\right)^{\prime} \\
0 & 0 & \left(m_{B}^{1}(0)\right)^{\prime} & \left(m_{B}^{2}(0)\right)^{\prime} & 0 & 0
\end{array}\right] .
\end{aligned}
$$

where $\mathbf{B}_{h u b}=-\mathbf{A} \tilde{\mathbf{u}}_{h u b} \overline{\mathbf{G}}$ (see Eq. (34)).

Moreover, based on Eq.(22), the value for the shape function at the hub $\left(\mathbf{S}_{h u b}=\mathbf{S}(0)\right)$ is defined as follows:

$$
\mathbf{S}(0)=\left[\begin{array}{cccccc}
m_{X}^{1}(0) & 0 & 0 & 0 & 0 & 0 \\
0 & 0 & m_{B}^{1}(0) & m_{B}^{2}(0) & 0 & 0 \\
0 & 0 & 0 & 0 & m_{B}^{1}(0) & m_{B}^{2}(0)
\end{array}\right]
$$

Finally, the contribution of wind excitation could be defined after specifying $\mathbf{Q}_{\text {wind }}$ components corresponding to the forces and the moments:

$$
\left(\mathbf{Q}^{w}\right)_{\bullet}^{T}=\mathbf{Q}_{\bullet}^{F}+\mathbf{Q}_{\bullet}^{M}
$$

where $\bullet=\{R, \theta, f\}$. Finally, the external load due to the wind loads will be:

$$
\mathbf{Q}_{e x t}^{w}=\left[\begin{array}{lll}
\left(\mathbf{Q}^{w}\right)_{R}^{T}, & \left(\mathbf{Q}^{w}\right)_{\theta}^{T}, & \left(\mathbf{Q}^{w}\right)_{f}^{T}
\end{array}\right]^{T}
$$




\subsection{Generator loads}

The more detailed derivation related to the sec. 2.6.2 is presented here. The force and moment components of the wind loads are defined as follows:

$$
\begin{aligned}
& \mathbf{Q}_{R}^{M}=0, \\
& \mathbf{Q}_{\theta}^{M}=\mathbf{T}^{T}+\mathbf{T}^{T} \mathbf{B}_{g e n}, \\
& \mathbf{Q}_{f}^{M}=\mathbf{T}^{T} \mathbf{A}\left[\begin{array}{cccccc}
0 & m_{T}^{1}(L) & 0 & 0 & 0 & 0 \\
0 & 0 & 0 & 0 & -\left(m_{B}^{1}(L)\right)^{\prime} & -\left(m_{B}^{2}(L)\right)^{\prime} \\
0 & 0 & \left(m_{B}^{1}(L)\right)^{\prime} & \left(m_{B}^{2}(L)\right)^{\prime} & 0 & 0
\end{array}\right] .
\end{aligned}
$$

where $\mathbf{B}_{\text {gen }}=-\mathbf{A} \tilde{\overline{\mathbf{u}}}_{\text {gen }} \overline{\mathbf{G}}$ (see Eq. (34)).

Finally, the external load contributed from the generator will be:

$$
\mathbf{Q}_{e x t}^{w}=\left[\left(\mathbf{Q}^{G}\right)_{R}^{T}, \quad\left(\mathbf{Q}^{G}\right)_{\theta}^{T}, \quad\left(\mathbf{Q}^{G}\right)_{f}^{T}\right]^{T}
$$

\subsection{Quadratic velocity force vector}

The quadratic velocity vector consists of centrifugal, gyroscopic, and Coriolis force components, which are considered as velocity-dependent inertia forces. One can also obtain this vector by using the virtual work of the inertia forces. The full derivation of the arguments in quadratic velocity vector is presented in [22], which is based on Lagranges equations. The velocity force vector is updated in each time step of the numerical integration procedure. Deriving closed-form expression for the quadratic velocity force vector in terms of generalized vector $\mathbf{q}$ is shown as follows:

$$
\mathbf{Q}_{V}=\left[\left(\mathbf{Q}_{V}\right)_{R}^{T},\left(\mathbf{Q}_{V}\right)_{\theta}^{T},\left(\mathbf{Q}_{V}\right)_{f}^{T}\right]^{T}
$$

where $\mathbf{Q}_{V}$ is the quadratic velocity vector which contains the gyroscopic, centrifugal, and Coriolis force components. The subvectors $\left(\mathbf{Q}_{V}\right)_{R}$ and $\left(\mathbf{Q}_{V}\right)_{\theta}$ are the velocity-dependent inertia forces associated, respectively, with the translational and rotational coordinates of the selected body coordinate system, and $\left(\mathbf{Q}_{V}\right)_{f}$ is the vector of velocity-dependent inertia forces associated with the elastic generalized coordinates of the body.

$$
\begin{aligned}
& \text { Subvector }\left(\mathbf{Q}_{V}\right)_{R},\left(\mathbf{Q}_{V}\right)_{\theta},\left(\mathbf{Q}_{V}\right)_{f} \\
& \left(\mathbf{Q}_{V}\right)_{R}=\dot{\mathbf{A}}\left[\int_{V^{i}} \rho \tilde{\overline{\mathbf{u}}} d V^{i}\right] \overline{\boldsymbol{\omega}}+\mathbf{A}\left[\int_{V^{i}} \rho \dot{\tilde{\mathbf{u}}} d V^{i}\right] \bar{\omega}+\mathbf{A}\left[\int_{V^{i}} \rho \tilde{\overline{\mathbf{u}}} d V^{i}\right] \dot{\overline{\mathbf{G}}} \dot{\theta}-\dot{\mathbf{A}}\left[\int_{V^{i}} \rho \dot{\overline{\mathbf{u}}} d V^{i}\right],
\end{aligned}
$$

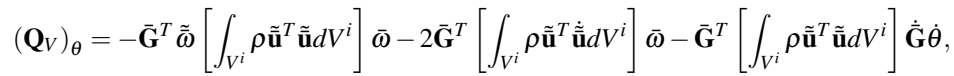

$$
\begin{aligned}
& \left(\mathbf{Q}_{V}\right)_{f}=\left[\int_{V^{i}} \rho \mathbf{S}^{T} \tilde{\tilde{\omega}} \tilde{\tilde{\mathbf{u}}} d V^{i}\right] \overline{\boldsymbol{\omega}}-2\left[\int_{V^{i}} \rho \mathbf{S}^{T} \dot{\tilde{\mathbf{u}}}^{T} d V^{i}\right] \overline{\boldsymbol{\omega}}-\left[\int_{V^{i}} \rho \mathbf{S}^{T} \tilde{\mathbf{u}}^{T} d V^{i}\right] \dot{\overline{\mathbf{G}} \dot{\theta}} .
\end{aligned}
$$

\section{References}

[1] Lim, H. T., Jeong, W. B., and Kim, K. J., Development of a wind turbine drive train engineering model, International Journal of Precision Engineering and Manufacturing, 2010, 11(3): pp. 407-417.

[2] Wasfy, T., and Noor, A., Computational Strategies for Flexible Multibody Systems, Applied Mechanics Review., 2003, 56(6): pp. 553-613.

[3] Mayo, J., Shabana, A.A., Dominguez, J., Geometrically nonlinear formulations of beams in flexible multibody dynamics Journal of Vibration Acoustic, 1995, 117(4): pp. 501-509. 
[4] Qin, D., J, Wang., T. C, Lim., Flexible Multibody Dynamic Modeling of a Horizontal Wind Turbine Drive train System ASME. J. Mech. Des., 2009, 131(11): pp. 114501-114509. DOI: 10.1115/1.3211094.

[5] Lee, D., D. H. Hodges., M. J, Patil., Multi-flexible-body Dynamic Analysis of Horizontal Axis Wind Turbines, Wind Energy., 2002, 5: pp. 281-300. DOI: 10.1002/we.66.

[6] Torstensson, P., Nielsen, J., Simulation of dynamic vehicle-track interaction on small radius curves Vehicle System Dynamics, 2011, 49(11): pp. 1711-1732, DOI 10.1080/00423114.2010.499468.

[7] Nada, A., Hussein, B., Megahed, S., Shabana, A., Use of the floating frame of reference formulation in large deformation analysis: experimental and numerical validation., Journal of Multi-Body Dynamics., 2014, 224(1): pp. 45-58.

[8] Den Dekker, H. A. D., Efficient Modeling of Rotational Effects for Wind Turbine Structural Dynamic Analysis:. (Masters Thesis). Delft University of Technology. Retrieved from http://resolver.tudelft.nl/uuid:68bcb8ff-a55b-4552-b351$8 f d c 4 f b 0 b 2432010$.

[9] Holm-Jorgensen, K., Nonlinear multibody dynamics of wind turbines. PhD Dissertation. Aalborg University, The Faculty of Engineering and Science, Division of Structural Mechanics, 2009.

[10] Shabana, A., Definition of the Slopes and the Finite Element Absolute Nodal Coordinate Formulation, Multibody System Dynamics., 1997, 1(3): pp. 339-348.

[11] Shabana, A., Flexible Multibody Dynamics: Review of Past and Recent Developments, Multibody System Dynamics, 1997, 1: pp. 189-222.

[12] Le. T., Battini, J., Hjiaj, M., Efficient formulation for dynamics of corotational 2D beams, Computational Mechanics, 2011, 48(2): pp. 153-161.

[13] Le. T., Battini, J., Hjiaj, M., A consistent 3D corotational beam element for nonlinear dynamic analysis of flexible structures, Computer Methods in Applied Mechanics and Engineering, 2014, 269, pp: 538-565.

[14] Wu, L. Tiso, P., Nonlinear model order reduction for flexible multibody dynamics: a modal derivatives approach, Multibody System Dynnamics, 2015, 36(4): pp. 405-425, DOI 10.1007/s11044-015-9476-5.

[15] Mayo, J., Domnguez, J., Geometrically non-linear formulation of flexible multibody systems in terms of beam elements: Geometric stiffness, Comput. Struct, 1996, 59(6): pp. 1039-1050.

[16] Sharf, I., Nonlinear strain measures, shape functions and beam elements for dynamics of flexible beams. Multibody System Dynamics, 1999, 3: pp. 189-205.

[17] Peeters, J., Simulation of Dynamic Drive Train Loads in a Wind Turbines. PhD Dissertation. Katholieke Universitet Leuven, 2006, Faculteit Ingenieurswetenshappen Departement Werktuigkunde Afdeling Productietechnieken Machinebouw en Automatisering Celestijnenlaan, Belgium.

[18] Bakr, E.M., Shabana, A.A.: Geometrically nonlinear analysis of multibody systems. Comput. Struct. 1986, 23(6), pp. 739-751.

[19] Rizzi, S.A., Przekop, A.: System identification-guided basis selection for reduced-order nonlinear response analysis. J. Sound Vib, 2008, 315(3), pp. 467-485.

[20] Schwertassek, R., Wallrapp, O., Shabana, A. Flexible multibody simulation and choice of shape functions. Nonlinear Dyn, 1999, 20(4), pp. 361-380.

[21] Schwertassek, R., Dombrowski, S., Wallrapp, O. Modal representation of stress in flexible multibody simulation. Nonlinear Dyn, 1999, 20(4), pp. 381-399.

[22] Sherif, K., K, Nachbagauer., A Detailed Derivation of the Velocity-Dependent Inertia Forces in the Floating Frame of Reference Formulation, ASME. J. Comput. Nonlinear Dynam., 2014, 9(4): pp. 281-300. DOI: 10.1115/1.4026083.

[23] Shabana, A,. Dynamics of Multibody Systems, Cambridge Press, New York, 2013.

[24] Singla, Puneet. Mortari, D. Junkins, J L., How to avoid singularity for Euler angle set, Advances in the Astronautical Sciences, 2005, 119: pp. 1409-1426. 
[25] Alipour, A. Zareian, F., Study Rayleigh Damping in Structures; Uncertainties and Treatments, World Conference on Earthquake Engineering, 2008.

[26] Adhikari, S., Damping Models for Structural Vibration, Cambridge University Engineering Department, Dissertation at the University of Cambridge for the Degree of Doctor of Philosophy, 2000.

[27] Ganander. H. The use of a code-generating system for the derivation of the equations for wind turbine dynamics. Wind Energy, 2003, 6(4): pp. 333-345.

[28] Xu, L.X., Yang, Y.H., Li, Y.G., et al. Modeling and analysis of planar multibody systems containing deep groove ball bearing with clearance, Mech. Mach. Theory, 2012, 56: pp. 69-88.

[29] Rolling bearings (general catalogue published by SKF group), 2012.

[30] Harris, T.A.. Rolling Bearing Analysis. John Wiley and Sons, Inc., fourth edition, 2001.

[31] Kotzalas, M. and Doll, G. Tribological advancements for reliable wind turbine performance. Phil. Trans. R. Soc, 2010, A 368: pp. 4829-4850.

[32] Johansson, H., Berbyuk, V. Statistical analysis of fatigue loads in a direct drive wind turbine. EWEA 2017 Barcelona Conference, 2010, A 368: pp. 4829-4850.

[33] International electrotechnical commission (IEC) Standard. 61400 Wind turbines part 1: Design requirements, 2005. 